\title{
How precise is the finite sample approximation of the asymptotic distribution of realised variation measures in the presence of jumps?
}

\author{
Almut E. D. Veraart
}

School of Economics and Management

Aarhus University

Bartholins Allé 10, Building 1322, DK-8000 Aarhus C

Denmark 


\title{
How precise is the finite sample approximation of the asymptotic distribution of realised variation measures in the presence of jumps?
}

\author{
Almut E. D. Veraart* \\ CREATES \\ School of Economics and Management \\ Aarhus University \\ DK-8000 Aarhus C \\ Denmark \\ Email: averaartecreates.au.dk
}

September 18, 2010

\begin{abstract}
This paper studies the impact of jumps on volatility estimation and inference based on various realised variation measures such as realised variance, realised multipower variation and truncated realised multipower variation. We review the asymptotic theory of those realised variation measures and present a new estimator for the asymptotic 'variance' of the centered realised variance in the presence of jumps. Next, we compare the finite sample performance of the various estimators by means of detailed Monte Carlo studies where we study the impact of the jump activity, the jump size of the jumps in the price and the presence of additional independent or dependent jumps in the volatility on the finite sample performance of the various estimators. We find that the finite sample performance of realised variance, and in particular of the log-transformed realised variance, is generally good, whereas the jump-robust statistics turn out not to be as jump robust as the asymptotic theory would suggest in the presence of a highly active jump process.

In an empirical study on high frequency data from the Standard \& Poor's Depository Receipt (SPY), we investigate the impact of jumps on inference on volatility by realised variance in practice.
\end{abstract}

Keywords: Realised variance; realised multipower variation; truncated realised variance; inference; stochastic volatility; jumps.

JEL codes: C10, C14, C58, G10.

\footnotetext{
*Financial support by the Center for Research in Econometric Analysis of Time Series, CREATES, funded by the Danish National Research Foundation, is gratefully acknowledged.
} 


\section{Introduction}

Volatility estimation and inference is one of the key problems in finance and has become one of the most active research areas in financial econometrics in the last decade. In particular, the availability of high frequency financial data makes it possible to construct various non-parametric volatility estimators, whose theoretical behaviour is now very well understood, see Andersen, Bollerslev, Diebold \& Labys (2001), Barndorff-Nielsen \& Shephard (2002, 2004a,b, 2007). Besides the question of estimating volatility, the recent literature has also addressed the problem of testing for jumps in asset price data, see Aït-Sahalia \& Jacod (2009b), Barndorff-Nielsen \& Shephard (2006), Huang \& Tauchen (2005), Lee \& Mykland (2008). In this paper, we address a closely related questions, which has not been studied empirically before: How do jumps impact inference on volatility based on various realised variation measures?

In order to answer that question, we will first focus on the most widely used and probably best understood non-parametric stochastic volatility estimator, i.e. on realised variance, see Andersen, Bollerslev, Diebold \& Ebens (2001), Barndorff-Nielsen \& Shephard (2002). Daily realised variance is defined as the sum of the squared intra-day returns. It can be shown, see Protter (2004), that this quantity is a consistent estimator for the quadratic variation of the price process, which is often regarded as a measure of accumulated daily variance. However, in order to make inference on the quadratic variation of the price process, we need a central limit result for the volatility estimator. Recently, Jacod (2008) has established a comprehensive limit theory for realised variance (among other functionals of semimartingales) when the logarithmic asset price is assumed to be an Itô semimartingale; the corresponding feasible limit theory has then been derived in Veraart (2010) and we refer to these two papers for details on the underlying theory.

In this paper, we present a new estimator for the jump part of the asymptotic 'variance' of the centered realised variance which is based on the threshold concept.

Next, this paper focuses on practical aspects of the new central limit theory for realised variance when the asset price exhibits jumps. First of all, we study the finite sample performance of the new theory by means of Monte Carlo studies. In particular, we will see that the feasible limit theory makes use of spot volatility estimators. Hence we compare in our simulation experiment the finite sample performance based on two widely used spot volatility estimators: locally average realised bipower variation and locally averaged truncated realised variance. Furthermore, we will see that the asymptotic normality of realised variance in the presence of jumps only holds when the asset price and the volatility do not jump together. In fact, it is now possible to test whether there are co-jumps in price and volatility, see Jacod \& Todorov (2010). However, from a practical point of view one might want to ask the question, how big the estimation error is if one uses the standard inference technique even if there are common jumps in the price and the volatility. This is studied in another simulation experiment in this paper.

Since economists are often interested in inference on the continuous part of the quadratic variation, we also study the impact of jumps on realised multipower and truncated realised variation measures, which are known to be robust to jumps. We review the asymptotic theory of these quantities briefly and include them into our Monte Carlo study. While such measures turn out to be robust to rare jumps, they are severely challenged — in finite samples — by the presence of a very active jump process generating many small jumps in addition to some bigger jumps.

Next, we carry out a small empirical study based on high frequency data from the Standard \& Poor's Depository Receipt (SPY) over a ten year period from 1998 to 2008. In this study, we investigate the impact of jumps on inference based on realised variance empirically. We estimate the quadratic variation of the asset price by realised variance and compute the corresponding confidence 
bounds using the new feasible limit theory. The main aim is to find out how the size of the confidence bounds differs depending on whether we account for jumps or not.

The remaining part of the paper is structured as follows. Section 2 reviews the asymptotic properties of realised variance, realised multipower variation and truncated realised multipower variation in the presence of jumps. Section 3 presents consistent estimators for the asymptotic 'variances' of centered realised variation measures. Here we also present a new estimator of the jump part of the asymptotic 'variance' of centered realised variance. In Section 4, we check the finite sample performance of the asymptotic theory, where we study the impact of jumps in the price and also the impact of co-jumps in the price and in the volatility on inference for realised variance. Next, we study the impact of jumps on the size of the confidence bounds for realised variance empirically in Section 5. Finally, Section 6 concludes. All technical assumptions, proofs and tables from the simulation study are relegated to the Appendix, see Sections A, B and C, respectively.

\section{Asymptotic theory for realised variation measures in the presence of jumps}

Recent research on nonparametric volatility estimation has focused on the class of Itô semimartingales as a model for the logarithmic asset price. In this section we will review the basic traits of Iô semimartingales and we will present some of the most often used nonparametric volatility estimators.

The focus will be on a non-technical presentation of the underlying theory and we refer to Jacod (2008) and Veraart (2010) for the technical details.

\subsection{The Itô semimartingale framework}

Let $\left(\Omega, \mathcal{A},\left(\mathcal{F}_{t}\right)_{t \geq 0}, \mathbb{P}\right)$ denoted a filtered probability space, on which we define a real-valued Itô semimartingale $X=\left(X_{t}\right)_{t \geq 0}$. Note that an Itô semimartingale is a semimartingale whose characteristics are absolutely continuous with respect to the Lebesgue measure. That means that it can be written as

$$
X_{t}=X_{0}+\int_{0}^{t} b_{s} d s+\int_{0}^{t} \sigma_{s} d W_{s}+J_{t}
$$

where $W=\left(W_{t}\right)_{t \geq 0}$ is a Brownian motion and $J=\left(J_{t}\right)_{t \geq 0}$ is a pure jump process defined by

$$
J_{t}=\int_{0}^{t} \int_{E} \kappa(\delta(s, x))(\mu-\nu)(d s, d x)+\int_{0}^{t} \int_{E} \kappa^{\prime}(\delta(s, x)) \mu(d s, d x),
$$

where $\mu$ is a Poisson random measure on $\mathbb{R}_{+} \times E$ with $(E, \mathcal{E})$ being an auxiliary space on the probability space $\left(\Omega, \mathcal{A},\left(\mathcal{F}_{t}\right)_{t \geq 0}, \mathbb{P}\right)$. Further, $\nu$ denotes the predictable compensator of $\mu$ and is given by $\nu(d s, d x)=d s \otimes \lambda(d x)$, where $\lambda$ is a $\sigma$-finite measure on $(E, \mathcal{E})$. We denote by $\kappa$ a truncation function which is bounded, has compact support and $\kappa(x)=x$ in a neighbourhood of 0 and is assumed to be continuous. Then we define $\kappa^{\prime}(x)=x-\kappa(x)$. The coefficients $b, \sigma$ and $\delta$ are assumed to satisfy some mild integrability conditions, see the Appendix for more details.

Let us now assume that the logarithmic asset price, denoted by $X=\left(X_{t}\right)_{t \geq 0}$, is modelled by such an Itô semimartingale. Clearly, this is a very general modelling framework since it allows for stochastic volatility, fairly general jumps and can account for the leverage effect, i.e. the usually negative correlation between asset prices and volatility, see Black (1976), Nelson (1991), since $\sigma$ and $W$ are not assumed to be independent. 
Our aim is now to focus on the volatility of the logarithmic asset price $X$ given by (1). A quantity which is closely related to the volatility of the logarithmic asset price, is the quadratic variation of $X$, which is given by

$$
[X]_{t}=\int_{0}^{t} \sigma_{s}^{2} d s+\sum_{0 \leq s \leq t}\left(\Delta X_{s}\right)^{2}
$$

where $\Delta X_{s}=X_{s}-X_{s-}$ denotes the jump of $X$ at time $s$, see Protter (2004) for more details. We see that the quadratic variation contains two parts: the continuous part given by the integrated squared volatility process $\sigma$ and the jump part, which consists of the sum of the squared jumps of $X$. From an economic point of view, the quadratic variation can be interpreted as a measure of the accumulated instantaneous conditional variance of the continuous and the jump part of the price over a time interval $[0, t]$, where $t$ is usually taken to be one day.

\subsection{Realised variance}

The key question is: How can we estimate this accumulated daily price variation, defined by (3), given high frequency financial data?

Assume that we observe the process $X$ over an interval $[0, T]$ for $T>0$ at times $i \Delta_{n}$ for $\Delta_{n}>0$ and $i=0, \ldots\left\lfloor T / \Delta_{n}\right\rfloor$. Further, let $0<t \leq T$. Note that we will always assume that $\Delta_{n} \rightarrow 0$ as $n \rightarrow \infty$. Typically we have $\Delta_{n}=1 / n$. So for its discretely observed increments we write

$$
\Delta_{i}^{n} X=X_{i \Delta_{n}}-X_{(i-1) \Delta_{n}} .
$$

Now we define the realised variance (RV) over the interval $[0, t]$ as the sum of the squared increments, i.e.

$$
R V_{t}^{n}=\sum_{i=1}^{\left\lfloor t / \Delta_{n}\right\rfloor}\left(\Delta_{i}^{n} X\right)^{2}
$$

It is well-known, see e.g. Andersen, Bollerslev, Diebold \& Ebens (2001), Barndorff-Nielsen \& Shephard (2002) and Protter (2004), that the realised variance estimates the quadratic variation of the underlying process consistently, i.e.

$$
R V_{t}^{n} \stackrel{u c p}{\longrightarrow}[X]_{t}, \quad \text { as } n \rightarrow \infty,
$$

where the convergence is uniform on compacts in probability (ucp), see Protter (2004, p. 57). Realised variance is now a widely used 'volatility' estimator, see e.g. Huang \& Tauchen (2005) and Andersen et al. (2007). However, in order to assess the quality of this estimator, one has to construct confidence intervals and, hence, one needs a central limit result for realised variance. From recent work by Jacod (2008, Theorem 2.12 (ii)), we obtain a central limit theory for realised variance in the presence of jumps.

Assume that Assumption (L-2), see Appendix, holds. Then

$$
\frac{1}{\sqrt{\Delta_{n}}}\left(\sum_{i=1}^{\left\lfloor t / \Delta_{n}\right\rfloor}\left(\Delta_{i}^{n} X\right)^{2}-[X]_{t}\right)=\frac{1}{\sqrt{\Delta_{n}}}\left(\sum_{i=1}^{\left\lfloor t / \Delta_{n}\right\rfloor}\left(\Delta_{i}^{n} X\right)^{2}-\left(\int_{0}^{t} \sigma_{s}^{2} d s+\sum_{0 \leq s \leq t}\left(\Delta X_{s}\right)^{2}\right)\right)
$$


converges, as $\Delta_{n} \rightarrow 0$, finite dimensionally stably in law to a random variable which has zero mean and (conditional) asymptotic 'variance' given by

$$
V_{t}:=2 I_{t}+2 D_{t}, \quad \text { where } \quad I_{t}:=\int_{0}^{t} \sigma_{s}^{4} d s, \quad D_{t}:=\sum_{p: T_{p} \leq t}\left(\sigma_{T_{p}-}^{2}+\sigma_{T_{p}}^{2}\right)\left(\Delta X_{T_{p}}\right)^{2},
$$

where $\left(T_{p}\right)_{p \geq 1}$ denote stopping times which are the enumerations of the jump times of $X$.

Remark Note that stable convergence in law is a stronger convergence concept than convergence in law, see Rényi (1963) and Jacod (2008) for more details. In particular, it will allow us to replace the asymptotic 'variance' $V_{t}$ by a consistent estimator $\widehat{V}_{t}$ without having an impact on the validility of the central limit theorem, see below.

Under the additional assumption that $X$ and $\sigma$ do not have common jumps, the above result simplifies to

$$
\frac{1}{\sqrt{\Delta_{n}}}\left(\sum_{i=1}^{\left\lfloor t / \Delta_{n}\right\rfloor}\left(\Delta_{i}^{n} X\right)^{2}-\left(\int_{0}^{t} \sigma_{s}^{2} d s+\sum_{0 \leq s \leq t}\left(\Delta X_{s}\right)^{2}\right)\right) \rightarrow M N\left(0, V_{t}\right),
$$

finite dimensionally stably in law. In this case, $D_{t}$ simplifies to $2 \sum_{p: T_{p} \leq t} \sigma_{T_{p}}^{2}\left(\Delta X_{T_{p}}\right)^{2}$, since the jump times of $X$ are not jump times of $\sigma$ and, hence, $\sigma_{T_{p}-}^{2}=\sigma_{T_{p}}^{2}$ for $p \in \mathbb{N}$ such that $T_{p} \leq t$. Note that $M N$ stands for mixed normal.

Remark When there are no jumps, the limit result is well-known, see e.g. Jacod (1994), Jacod \& Protter (1998) and Barndorff-Nielsen \& Shephard (2002).

In order to use this limit result in practice, we need a consistent estimator for the asymptotic 'variance' $V_{t}$.

Let us assume that $\widehat{V}_{t}^{n}$ denotes such a consistent estimator of $V_{t}$, then we get

$$
\frac{1}{\sqrt{\Delta_{n} \widehat{V}_{t}^{n}}}\left(\sum_{i=1}^{\left\lfloor t / \Delta_{n}\right\rfloor}\left(\Delta_{i}^{n} X\right)^{2}-\left(\int_{0}^{t} \sigma_{s}^{2} d s+\sum_{0 \leq s \leq t}\left(\Delta X_{s}\right)^{2}\right)\right) \rightarrow N(0,1),
$$

where the convergence is in law.

Remark Since the central limit result (5) holds stably in law (and not just in law), we can replace $V_{t}$ by a consistent estimator $\widehat{V}_{t}^{n}$ and obtain the feasible central limit result (6) without further proof.

\subsection{Realised multipower variation}

We have seen that the realised variance estimates the entire quadratic variation consistently. However, it is also interesting to study the continuous part and the jump part of the qudratic variation separately. In order to do that, two concepts have been proposed: realised multipower variation, see Barndorff-Nielsen \& Shephard (2004b, 2006), and truncated realised variation, see Mancini (2001). More recently, a combination of these two concept has also been studied, see Corsi et al. (2010). Here, we will review these concepts briefly.

The concept of realised multipower variation (RMPV) has been introduced by Barndorff-Nielsen $\&$ Shephard $(2004 b, 2006)$ as a consistent estimator of the continuous part of the quadratic variation, 
which we denote by $[X]_{t}^{c}=\int_{0}^{t} \sigma_{s}^{2} d s$. Subsequently, it has also been studied by Woerner (2006), Jacod (2006).

In the following, we will denote by $\mu_{x}=\mathbb{E}\left(|U|^{x}\right)=\pi^{-1 / 2} 2^{x / 2} \Gamma\left(\frac{x+1}{2}\right)$, for $U \sim N(0,1)$ and $x>0$ the $x$ th absolute moment of a standard normally distributed random variable. Further, let $I \in \mathbb{N}$ and let $\mathbf{r}=\left(r_{1}, \ldots, r_{I}\right)$ denote a multi-index where $r_{i}>0$ for $i=1, \ldots, I$. We write

$$
|\mathbf{r}|=\sum_{i=1}^{I} r_{i}, \quad \mathbf{r}_{+}=\max _{i \in 1, \ldots, I} r_{i}, \quad \mathbf{r}_{-}=\min _{i \in 1, \ldots, I} r_{i}, \quad \mu_{\mathbf{r}}=\prod_{i=1}^{I} \mu_{r_{i}} .
$$

We define the realised multipower variation (RMPV) by

$$
R M P V(\mathbf{r})_{t}^{n}=\Delta_{n}^{1-|\mathbf{r}| / 2} \sum_{i=1}^{\left\lfloor t / \Delta_{n}\right\rfloor} \prod_{j=1}^{I}\left|\Delta_{i+j-1}^{n} X\right|^{r_{j}} .
$$

From Jacod (2006), we know that under assumption (H), see Appendix, and if $\mathbf{r}_{+}<2$, then

$$
R M P V(\mathbf{r})_{t}^{n} \stackrel{u c p}{\rightarrow} \mu_{\mathbf{r}} \int_{0}^{t} \sigma_{s}^{|\mathbf{r}|} d s, \quad \text { as } \Delta_{n} \rightarrow 0 .
$$

The corresponding central limit result only holds under stricter conditions (mainly on the jump part of $X$ ), see Jacod (2006). Under assumption (A), see Appendix, which basically says that the jump part of $X$ is of finite variation and the volatility process $\sigma$ never vanishes, then for $\mathbf{r}_{+}<1$ :

$$
\frac{1}{\sqrt{\Delta_{n}}}\left(R M P V(\mathbf{r})_{t}^{n}-\mu_{\mathbf{r}} \int_{0}^{t} \sigma_{s}^{|\mathbf{r}|} d s\right) \rightarrow M N\left(0, A(\mathbf{r}) \int_{0}^{t} \sigma_{s}^{2|\mathbf{r}|} d s\right)
$$

where the convergence is stably in law and

$$
A(\mathbf{r})=\prod_{i=1}^{I} \mu_{2 r_{i}}-(2 I-1) \prod_{i=1}^{I} \mu_{r_{i}}^{2}+2 \sum_{i=1}^{I-1} \prod_{j=1}^{i} \mu_{r_{j}} \prod_{j=I-i+1}^{I} \mu_{r_{j}} \prod_{j=1}^{I-i} \mu_{r_{j}+r_{j+i}} .
$$

Remark Note that, in the presence of jumps, the above central limit theorem does not hold for the realised bipower variation, where $I=2$ and $r_{1}=r_{2}=1$. See Vetter (2010) for a detailed treatment of that case.

The choices $|\mathbf{r}|=2$ and $|\mathbf{r}|=4$ are of particular interest in financial econometrics, since they lead to consistent estimators of integrated variance and integrated quarticity, respectively. In many applications, we are interested in the case where $r_{i}=c / I$ for $c \in \mathbb{N}$ and $i=1, \ldots, I$. In this case, $A(\mathbf{r})$ simplifies to

$$
A((c / I, \ldots, c / I))=\mu_{2 c / I}^{I}-(2 I-1) \mu_{c / I}^{2 I}+2 \sum_{i=1}^{I-1} \mu_{c / I}^{2 i} \mu_{2 c / I}^{I-i} .
$$

If $c=2, I=2$, we obtain realised bipower variation and for $c=2, I=3$ realised tripower variation. More generally, for estimating the integrated $|\mathbf{r}|$ th power of $\sigma$, we could use any multipower variation (satisfying the assumptions above) such that $c=|\mathbf{r}| / I$. However, from the formulae above we see that the efficiency of multipower variation decreases as $I \rightarrow \infty$, see also Table 1 for some examples.

The main intuition behind the concept of realised multipower variation lies in the fact that the probability of having several jumps in neighbouring intervals is very low, hence the effect of jumps is averaged out compared to realised variance, say. 


\begin{tabular}{c|ccc}
\hline$c, I$ & $\mu_{c / I}$ & $\mu_{\mathbf{r}}^{-1}=\mu_{c / I}^{-I}$ & $\mu_{\mathbf{r}}^{-2} A(\mathbf{r})$ \\
\hline$c=2, I=2$ & $\sqrt{2 / \pi} \simeq 0.797$ & $\pi / 2 \simeq 1.570$ & $\frac{1}{4} \pi^{2}+\pi-3 \simeq 2.608$ \\
$c=2, I=3$ & 0.802 & 1.935 & 3.061 \\
$c=4, I=3$ & 0.830 & 1.743 & 13.649 \\
$c=4, I=4$ & 0.797 & 2.467 & 14.916 \\
\hline
\end{tabular}

Table 1: Scaling and efficiency parameters for the realised multipower variation. Here we choose $\mathbf{r}=\left(r_{1}, \ldots, r_{I}\right)$, where $r_{i}=c / I$, for $c, I \in \mathbb{N}, i=1, \ldots, I$. Then $\lim _{I \rightarrow \infty} \mu_{\mathbf{r}}^{-2} A(\mathbf{r})=\pi^{2} c^{2} / 8$.

Remark In order to improve the finite sample performance of realised multipower, in particular when it is compared to realised variance, one often multiplies the realised multipower variation by the factor $\frac{\left\lfloor t / \Delta_{n}\right\rfloor}{\left\lfloor t / \Delta_{n}\right\rfloor-I+1}$, which converges to 1 as $n \rightarrow \infty$. This factor makes up for the fact that realised variance consists of $\left\lfloor t / \Delta_{n}\right\rfloor$ terms in the sum, whereas RMPV only consists of $\left\lfloor t / \Delta_{n}\right\rfloor-I+1$ terms.

Another adjusted version of RMPV, which one can often find are staggered versions, see e.g. Huang \& Tauchen (2005), which make RMPV robust towards some types of market microstructure effects.

\subsection{Truncated realised variance}

Besides the concept of realised multipower variation there is the concept of truncated RV or RMVP. The idea of truncating RV to only use 'small' increments and, hence, get rid of jumps has been introduced by Mancini (2001), see also Mancini (2009), and has subsequently been studied in a more general framework by Jacod (2008). The main results are, see Jacod (2008, Theorem 2.4 (iii) and Theorem 2.10), that the truncated realised variance (TRV) defined by

$$
T R V(\omega, \alpha)_{t}^{n}=\sum_{i=1}^{\left\lfloor t / \Delta_{n}\right\rfloor}\left(\Delta_{i}^{n} X\right)^{2} \mathbb{I}_{\left\{\left|\Delta_{i}^{n} X\right| \leq \alpha \Delta_{n}^{\omega}\right\}},
$$

for $\alpha>0$ and $\omega \in(0,1 / 2)$ is a consistent estimator of $[X]_{t}^{c}$, provided assumption $(\mathrm{H})$, see Appendix, holds. Further, under the stronger assumption (B) (on the activity index of the jumps in $X$ ), we have the following central limit theorem:

$$
\frac{1}{\sqrt{\Delta_{n}}}\left(T R V(\omega, \alpha)_{t}^{n}-\int_{0}^{t} \sigma_{s}^{2} d s\right) \rightarrow M N\left(0,2 \int_{0}^{t} \sigma_{s}^{4} d s\right), \quad \text { as } \Delta_{n} \rightarrow 0 .
$$

Remark When we compare RMPV(r) with $|\mathbf{r}|=2$ and TRV, we observe that both classes of estimators are consistent and asymptotically unbiased estimators for $[X]^{c}$. The advantage of the TRV estimator is that it is more efficient then $\operatorname{RMPV}(\mathbf{r})$. However, the nice feature of the RMPV(r) estimator lies in the fact that it does not require to choose a threshold, which can be complicated in practice.

\subsection{Truncated/threshold realised multipower variation}

Recently, the two concepts of multipower variation and truncation have been combined in order to get an estimator which achieves even better finite sample properties, see Corsi et al. (2010). Truncated 
realised multipower variation (TRMPV) is defined by

$$
\operatorname{TRMPV}(\mathbf{r}, \alpha, \omega)_{t}^{n}=\Delta_{n}^{1-|\mathbf{r}| / 2} \sum_{i=1}^{\left\lfloor t / \Delta_{n}\right\rfloor} \prod_{j=1}^{I}\left|\Delta_{i+j-1}^{n} X\right|^{r_{j}} \mathbb{I}_{\left\{\left|\Delta_{i+j-1}^{n} X\right| \leq \alpha_{j} \Delta_{n}^{\omega_{j}}\right\}}
$$

where we use the notation as in the RMPV case and, additionally, $\alpha=\left(\alpha_{1}, \ldots, \alpha_{I}\right)$ and $\omega=$ $\left(\omega_{1}, \ldots, \omega_{I}\right)$, where all $\alpha_{i}>0$ and $\omega_{i} \in(0,1 / 2)$ for $i=1, \ldots, I$. Regarding the consistency, we get the same result as in (8), where RMPV is replaced by TRMPV. Under the same assumptions as above, we also obtain a central limit theorem of the form (9), where RMPV is replaced by TRMPV. In addition, the central limit theorem also holds for the case that $\mathbf{r}_{+}=1$ and, hence, includes truncated realised bipower variation.

\subsection{Difference of RV and RMPV}

So far, we have seen that RV can be used for making inference on $[X]$ and RMPV, TRV and TRMPV can be used for inference on $\left[X^{c}\right]$. In order to make inference on the jump part of the quadratic variation, we can use the difference of RV and RMPV, which has been studied in Veraart (2010).

Under assumption (C), see Appendix, we have that for $r_{i}=2 / I$

$$
\frac{1}{\sqrt{\Delta_{n}}}\left(R V_{t}^{n}-\mu_{2 / I}^{-I} R M P V(\mathbf{r})_{t}^{n}\right) \rightarrow M N\left(0, \theta_{I} I_{t}+2 D_{t}\right), \quad \text { as } \Delta_{n} \rightarrow 0,
$$

where $\theta_{I}=\mu_{2 / I}^{-2 I} A((2 / I, \ldots, 2 / I))-2$.

\subsection{Final remarks regarding the asymptotic theory}

It should be noted that the asymptotic theory for the realised measures reviewed above has been derived under the assumption that there is always a Brownian component present, i.e. the price process does not solely consist of a pure jump component. For pure jump processes, the asymptotic theory is much less developed. However, for some subclasses of pure jump processes there are some asymptotic results for realised measures, see e.g. Jacod (2004), Todorov \& Tauchen (2010) for some first investigations along those lines. However, the assumption that a Brownian component is always present does not seem to be that unrealistic in the light of recent findings by Cont \& Mancini (2007) and Aït-Sahalia \& Jacod (2010).

\section{Consistent estimators of the asymptotic variance of centered realised variation measures}

In order to use the central limit results reviewed above to make inference on $[X],\left[X^{c}\right]$ or on $\left[X^{d}\right]$, we need a consistent estimators for the conditional asymptotic 'variances'. In order to do that, we proceed in two steps: We review the well-known estimators for the continuous part, i.e. for $I_{t}$, and then we construct a new estimator for the jump part $D_{t}$. 


\subsection{Estimating the continuous part}

From the previous section, we know that we can use RMPV, and in particular realised tripower variation with $r_{i}=4 / 3$, for estimating $I_{t}$, i.e. we define

$$
\hat{I}_{t}^{n}=\mu_{4 / 3}^{-3} R M P V((4 / 3,4 / 3,4 / 3))=\mu_{4 / 3}^{-3} \Delta_{n}^{-1} \sum_{i=1}^{\left\lfloor t / \Delta_{n}\right\rfloor-2}\left|\Delta_{i}^{n} X\right|^{4 / 3}\left|\Delta_{i+1}^{n} X\right|^{4 / 3}\left|\Delta_{i+2}^{n} X\right|^{4 / 3} .
$$

Then $\hat{I}_{t}^{n}$ is a consistent and unbiased estimator of the integrated quarticity even in the presence of jumps in the price process, in particular $\hat{I}_{t}^{n} \stackrel{u c p}{\longrightarrow} I_{t}=\int_{0}^{t} \sigma_{s}^{4} d s$, as $n \rightarrow \infty$. Other choices of $\operatorname{RMPV}(\mathbf{r})$ with $|\mathbf{r}|=4$ would be possible, too, but the choice above is the most efficient one from the RMPV(r) estimators which are robust towards jumps and are of the type $r_{i}=4 / I$.

\subsection{Estimating the jump part}

Next, we propose a new estimator for the jump process $D_{t}=\sum_{p: T_{p} \leq t}\left(\sigma_{T_{p}-}^{2}+\sigma_{T_{p}}^{2}\right)\left(\Delta X_{T_{p}}\right)^{2}$, which is based on the truncation concept. In particular, we use a localised version of TRV for estimating the spot volatility $\sigma^{2}$ and we use the truncation concept again, this time using the big increments only, for approximating the jumps of $D$. Altogether, we get the following result.

Proposition 3.1 Let assumption (G1), see Appendix, be satisfied. Let $\left(k_{n}\right)_{n \in \mathbb{N}} \subseteq \mathbb{N}$ such that $k_{n} \rightarrow$ $\infty$ and $k_{n} \Delta_{n} \rightarrow 0$ as $n \rightarrow \infty$. Further, let

$$
I_{n, t}(i)=\left\{j \in \mathbb{N}: j \neq i: 1 \leq j \leq\left\lfloor t / \Delta_{n}\right\rfloor,|i-j| \leq k_{n}\right\} .
$$

For $\alpha>0, \omega \in\left(0, \frac{1}{2}\right)$, we define

$$
\widehat{D}_{t}^{(1), n}:=\widehat{D}_{t}^{(1), n}(\alpha, \omega):=\frac{1}{k_{n} \Delta_{n}} \sum_{i=1}^{\left\lfloor t / \Delta_{n}\right\rfloor}\left(\Delta_{i}^{n} X\right)^{2} \mathbb{I}_{\left\{\left|\Delta_{i}^{n} X\right|>\alpha \Delta_{n}^{\omega}\right\}} \sum_{j \in I_{n, t}(i)}\left(\Delta_{j}^{n} X\right)^{2} \mathbb{I}_{\left\{\left|\Delta_{j}^{n} X\right| \leq \alpha \Delta_{n}^{\omega}\right\}} .
$$

Then $\widehat{D}_{t}^{(1), n} \stackrel{\mathbb{P}}{\rightarrow} D_{t}$, as $n \rightarrow \infty$.

Proof The proof is given in the appendix.

An alternative estimator of $D_{t}$ has recently been proposed in Veraart (2010, Theorem 2). Under assumption (D). Further, we define an index set $I^{*}(n, i)$ by

$$
I_{n, t}^{*}(i)=\left\{j \in \mathbb{N} \backslash\{i-1, i, i-1\}: 1 \leq j \leq\left\lfloor t / \Delta_{n}\right\rfloor,|i-j| \leq k_{n}\right\} .
$$

Then,

$$
\widehat{D}_{t}^{(2), n}=\max \left(\sum_{i=1}^{\left[t / \Delta_{n}\right]}\left(\Delta_{i}^{n} X\right)^{2} \frac{\mu_{1}^{-2}}{\left(k_{n}-2\right) \Delta_{n}} \sum_{j \in I^{*}(n, i)}\left|\Delta_{j}^{n} X\right|\left|\Delta_{j+1}^{n} X\right|-2 \hat{I}_{t}^{n}, 0\right)
$$

is also a consistent estimator of $D_{t}$. 


\subsection{Putting it together}

Recall that, for inference on the realised variance, we need to estimate $V_{t}=2 I_{t}+2 D_{t}$. Hence, we choose the following two estimators:

$$
\begin{aligned}
\widehat{V}_{t}^{(1), n}:=\widehat{V}_{t}^{(1), n}(\alpha, \omega) & =2 \hat{I}_{t}^{n}+2 \widehat{D}_{t}^{(1), n}(\alpha, \omega), \\
\widehat{V}_{t}^{(2), n} & =2 \hat{I}_{t}^{n}+2 \widehat{D}_{t}^{(2), n} .
\end{aligned}
$$

\subsection{Relevant quantities for the simulation study}

After we have reviewed the asymptotic theory for (truncated) RV and (truncated) RMPV in the presence of jumps, we study the finite sample properties of these estimators. In order to do that, we define the quantities of interest, which are asymptotically (under the appropriate assumptions) standard normally distributed.

Now we can combine the previous results and obtain a feasible central limit theory for realised variance in the presence of jumps, see (Veraart 2010, Corollary 5).

Let $t=1$ and $\Delta_{n}=1 / n$ and assume that $X_{0}=0$. Then $[X]_{1}=\int_{0}^{1} \sigma_{s}^{2} d s+\sum_{p: T_{p} \leq 1}\left(\Delta X_{s}\right)^{2}$. Let

$$
\begin{aligned}
T^{(1)}\left(k_{n}\right):=T^{(1)}\left(k_{n}, \alpha, \omega\right) & =\sqrt{n}\left(\sum_{i=1}^{n}\left(\Delta_{i}^{n} X\right)^{2}-[X]_{1}\right)\left(\widehat{V}_{1}^{(1), n}(\alpha, \omega)\right)^{-1 / 2} \\
T^{(2)}\left(k_{n}\right) & =\sqrt{n}\left(\sum_{i=1}^{n}\left(\Delta_{i}^{n} X\right)^{2}-[X]_{1}\right)\left(\widehat{V}_{1}^{(2), n}\right)^{-1 / 2}
\end{aligned}
$$

By applying the delta method for stable convergence, see Dette et al. (2006), to the logarithmic transform of the realised variance, we deduce that

$$
\begin{aligned}
\bar{T}^{(1)}\left(k_{n}\right) & :=\bar{T}^{(1)}\left(k_{n}, \alpha, \omega\right) \\
& =\sqrt{n}\left(\log \left(\sum_{i=1}^{n}\left(\Delta_{i}^{n} X\right)^{2}\right)-\log \left([X]_{1}\right)\right)\left(\widehat{V}_{1}^{(1), n}(\alpha, \omega)\right)^{-1 / 2} \sum_{i=1}^{n}\left(\Delta_{i}^{n} X\right)^{2}, \\
\bar{T}^{(2)}\left(k_{n}\right) & =\sqrt{n}\left(\log \left(\sum_{i=1}^{n}\left(\Delta_{i}^{n} X\right)^{2}\right)-\log \left([X]_{1}\right)\right)\left(\widehat{V}_{1}^{(2), n}\right)^{-1 / 2} \sum_{i=1}^{n}\left(\Delta_{i}^{n} X\right)^{2},
\end{aligned}
$$

are asymptotically standard normally distributed.

In the following simulation study, we will study the finite sample performance of these statistics. Furthermore, we can now construct asymptotic confidence intervals for the quadratic variation of the $\log$-price process. The corresponding upper and lower confidence bounds are hence given by

$$
\sum_{i=1}^{n}\left(\Delta_{i}^{n} X\right)^{2} \pm q_{1-\frac{\alpha}{2}} \sqrt{n^{-1} \widehat{V}_{1}^{(1), n}(\alpha, \omega)}, \quad \text { and } \quad \sum_{i=1}^{n}\left(\Delta_{i}^{n} X\right)^{2} \pm q_{1-\frac{\alpha}{2}} \sqrt{n^{-1} \widehat{V}_{1}^{(2), n}}
$$

respectively, where $q_{1-\frac{\alpha}{2}}$ corresponds to the $1-\frac{\alpha}{2}$ quantile of a standard normally distributed random variable. Analogously as above, we construct statistics based on suitably centered and scaled RMPV and TRMPV, see below for more details. 


\section{Simulation study}

After we have reviewed the feasible central limit theory for realised variation measures, we study their finite sample behaviour by carrying out several Monte Carlo experiments. In particular, we simulate data from seven different stochastic volatility models for various different jump specifications, see Section 4.2 for more details. Then we compute 20 different statistics consisting of suitably centered and scaled realised measures. More precisely, we compute the ten quantities defined in Section 4.3 and the corresponding log-transformed quantities (using the delta method for stable convergence).

\subsection{Research questions addressed in the simulation study}

The simulation is designed such that it addresses the following research questions regarding the finite sample performance of realised variance:

- The estimator $\widehat{D}_{t}$ of the jump part of the asymptotic 'variance' of centered RV makes use of spot volatility estimators. Here we use locally averaged realised bipower variation and truncated realised variance. Which estimator is more reliable? How shall we choose the local window $k_{n}$ (which has to satisfy $k_{n} \rightarrow \infty$ and $k_{n} \Delta_{n} \rightarrow 0$ as $n \rightarrow \infty$ ). We compare the choice of $k_{n}=\left\lfloor 50 \Delta_{n}^{-1 / 4}\right\rfloor$ as suggested by Ait-Sahalia \& Jacod (2009b) with the choice $k_{n}=\left\lfloor\Delta_{n}^{-1 / 2}\right\rfloor$ which is more in line with Lee \& Mykland (2008) who suggested to choose a relatively small local window so that the computational intensity does not become too high. Note that we use $\Delta_{n}=1 / n$ for $n \in\{39,78, \ldots, 23400\}$, hence $\left\lfloor\Delta_{n}^{-1 / 2}\right\rfloor \leq\left\lfloor 50 \Delta_{n}^{-1 / 4}\right\rfloor$.

- Which estimator for $D_{t}$ is the better one? The one derived in Veraart (2010) or the one presented in this paper?

- How good is the finite sample performance of realised variance in general?

- Does the finite sample performance change when the activity level of the jump process and/or the jump size increase? In the presence of common jumps in the price and the volatility, centered RV is not asymptotically normally distributed. How does this statistic behave in such a framework?

- How do the log-transformed quantities perform compared to the raw statistics?

Regarding realised multipower variation and truncated realised measures, we aim to shed some light on the following questions:

- How robust are RMPV and TRMV towards jumps of finite and infinite activity in finite samples?

- Which jump robust measure performs best in the presence of jumps?

\subsection{Simulation design}

In order to study the impact of jumps on the performance of realised variation measure, we simulate from a variety of stochastic volatility models with and without jumps.

Throughout the simulation study, we choose one day as basic unit of time for our simulation. We then simulate the diffusion part based on an Euler scheme, where we choose the increment of one second per tick on the Euler clock, where we have 23,400 seconds a day (which corresponds to the equity market being open for 6.5 hours a day). 
Diffusion (D): The Heston stochastic volatility model serves as a jump-free benchmark model where the choice of parameters is taken from the empirical results in Ait-Sahalia \& Kimmel (2007). I.e. $d X_{t}=\sigma_{t} d W_{t}, d \sigma_{t}^{2}=\kappa\left(\beta-\sigma_{t}^{2}\right) d t+\gamma \sigma_{t} d B_{t}, \mathbb{E}\left(d W_{t} d B_{t}\right)=\rho d t$, where $\beta^{1 / 2}=0.4, \gamma=$ $0.5, \kappa=5, \rho=-0.5$. See Table 3 for the results.

Jump diffusion (JD): Using the same model specification for the continuous part as in (D), we now add compound Poisson jumps with normally distributed jumps: $d X_{t}=\sigma_{t} d W_{t}+d \sum_{i=1}^{N_{t}} J_{i}$, where $N_{t}$ is a Poisson process with i.i.d. normally distributed jump sizes such that $\sum_{i=1}^{N_{t}} J_{i} \mid N_{t} \sim$ $N\left(0, \sigma_{J}^{2}\right)$. In fact, we work with a stratified compound Poisson process having 1,5 or 10 jumps per day which are uniformly distributed over the day. Also, when we have more jumps, we decrease their size, so that the contribution of the jump 'variance' stays the same throughout the simulation. In order to achieve that, we simulate the jump sizes conditional on $N_{t}=c$ from $J_{i} \sim N\left(0, \frac{\sigma_{J}^{2}}{c}\right)$. Note that, working with the stationary $\sigma^{2}$, we have that $\mathbb{E}\left(\int_{0}^{1} \sigma_{s}^{2} d s\right)=$ $\mathbb{E}\left(\sigma_{0}^{2}\right)=\beta, \mathbb{E}\left(\sum_{i=1}^{c} J_{i}^{2}\right)=c \operatorname{Var}\left(J_{1}\right)=\sigma_{J}^{2}$. We choose $\sigma_{J}^{2}$ such that $p=\frac{\sigma_{J}^{2}}{\beta+\sigma_{J}^{2}}$, i.e. the percentage of the mean of the jump part of the quadratic variation compared to the mean of the total variation equals $15 \%$ or $30 \%$. Note that empirical studies sometimes find a much lower jump contribution, see e.g. Huang \& Tauchen (2005) who found up to 7\%. See Tables 4, 5, 6 for the results.

Infinite activity jumps in the price (IAP): Now let $d X_{t}=\sigma_{t} d W_{t}+d L_{t}$, where $L$ is a symmetrical tempered $\alpha$-stable Lévy process for $0<\alpha<1$ and $\lambda>0$. I.e. the Lévy density of $L$ is given by $\nu(d x)=|x|^{-(\alpha+1)} e^{-\lambda x} d x$. Clearly, $\operatorname{Var}\left(L_{1}\right)=2 \Gamma(2-\alpha) / \lambda^{2-\alpha}$. As before, we want the percentage of the jump part of the variance to be $p=15 \%, 30 \%$, where $\operatorname{Var}\left(L_{1}\right)=\beta p /(1-p)$. Hence we choose $\lambda=\left(\frac{\beta p}{2(1-p)}\right)^{1 /(\alpha-2)}$ for $\alpha=0.1,0.5,0.9$. Note that the simulation of the tempered stable Lévy process is based on the series representation given in Rosinski (2001). See Tables 7, 8, 9 for the results.

Next, we study the case of having jumps in the volatility process.

Independent finite activity jumps in volatility and price (IFAJ): Here we have $d X_{t}=\sigma_{t} d W_{t}+$ $d J_{t}^{(1)}, d \sigma_{t}^{2}=\kappa\left(\beta-\sigma_{t}^{2}\right) d t+\gamma \sigma_{t} d B_{t}+d J_{t}^{(2)}, \mathbb{E}\left(d W_{t} d B_{t}\right)=\rho d t$, where $J^{(1)}$ and $J^{(2)}$ being independent stratified compound Poisson processes (as before) with 5 and 10 jumps per day. See e.g. Zhou (2003) and the references therein for the existence of a process $\sigma^{2}$ defined as above. The jump sizes of $J^{(1)}$ are drawn from a double exponential distribution with equal probability of positive and negative jumps and the jump sizes of the independent process $J^{(2)}$ are exponentially distributed. Again, the parameters are chosen such that the jump percentage of the total variation is set to $15 \%$. See Tables 10,11 for the results.

Common finite activity jumps in volatility and price (CFAJ): The simulation set up is similar to IFAJ, but in order to make the jump processes $J^{(1)}, J^{(2)}$ dependent, we work with the same jump times and, in particular, we set $\left|J^{(1)}\right|=J^{(2)}$, where we allow for 5 or 10 jumps a day. See Tables 12, 13 for the results.

BNS model with independent jumps in price and volatility (BNSIJ): Next, we focus on the BarndorffNielsen \& Shephard $(2001,2002)$ model, where the volatility process is purely jump-driven, in particular: $d X_{t}=\sigma_{t} d W_{t}+d J_{t}, d \sigma_{t}^{2}=-\lambda \sigma_{t}^{2} d t+d L_{\lambda t}$, where $L$ is an inverse Gaussian $(I G(\mu, l))$ subordinator with parameters $\lambda=1, \mu=0.16, l=\mu^{2} . J$ is a stratified Poisson 
process, independent of $L$, with normally distributed jumps (as in JD), with $0,1,5,10$ jumps a day. Again the percentage of the jump variation is set to $15 \%$. See Tables 14, 15, 16, 17 for the results.

BNS model with common jumps in price and volatility (BNSCJ): Finally, we simulate the classical BNS model with common IG jumps, i.e. $d X_{t}=\sigma_{t} d W_{t}+\rho d L_{\lambda t}, d \sigma_{t}^{2}=-\lambda \sigma_{t}^{2} d t+d L_{\lambda t}$, with the parameter choices as in BNSIJ and, further, $\rho \in\{-0.2,-0.4\}$. See Tables 18, 19 and Figure 5 for the results. Note that the parameter $\rho$ in the BNS model is not a correlation parameter as such (since it is not restricted to be in $[-1,1]$ ), but it plays its role, see e.g. Veraart \& Veraart (2010) for more details.

\subsection{Quantities of interest in the simulation study}

According to the asymptotic theory, properly centered and scaled (truncated) realised (multi-) power variation converge -under the assumptions stated above- to standard normally distributed random variables. In order to check their finite sample performance, we compute these suitably centered and normalised quantities, where in the tables the column with the heading R.V.M specifies, which realised variation measure is studied. Throughout the simulation study, we use the following abbreviations for the realised variance measures:

RV: Centered realised variance, scaled by $\widehat{V}_{1}^{(1), n}(\alpha, \omega)$, i.e. $T^{(1)}\left(k_{n}, \alpha, \omega\right)$;

RV2: Centered realised variance, scaled by $2 \hat{I}_{1}^{n}+2 \sum_{i}\left(\widehat{\sigma}_{(i-1) \Delta_{n}}^{2}+\widehat{\sigma}_{(i-1) \Delta_{n}-}^{2}\right)\left(\Delta_{i}^{n} X\right) \mathbb{I}_{\left\{\left|\Delta_{i}^{n} X\right|>\alpha \Delta_{n}^{\omega}\right\}}$, where the spot volatility is estimated by locally averaged realised bipower variation;

pRV: Centered realised variance, scaled by $2 \hat{I}_{1}^{n}+2 \max \left\{\sum_{i}\left(\Delta_{i}^{n} X\right)^{2}\left(\widehat{\sigma}_{(i-1) \Delta_{n}}^{2}+\widehat{\sigma}_{(i-1) \Delta_{n}-}^{2}\right)-\right.$ $\left.2 \hat{I}_{1}^{n}, 0\right\}$, where the spot volatility is estimated by locally averaged truncated realised variance;

pRV2: Centered realised variance, scaled by $\widehat{V}_{1}^{(2), n}$, i.e. $T^{(2)}\left(k_{n}\right)$.

cRV: Centered realised variance, scaled by the estimator of the continuous part of the asymptotic 'variance' only, i.e. by $2 \hat{I}_{1}^{n}$.

(Truncated) realised multipower variations:

TRV: Centered and scaled truncated realised variance;

TR2V: Centered and scaled truncated realised bipower variation;

R2V: Centered and scaled realised bipower variation;

R3V: Centered and scaled realised tripower variation;

R4V: Centered and scaled realised quadpower variation;

Note that the threshold parameters are chosen to be $\alpha=2, \omega=0.47$ following the simulation study in Ait-Sahalia \& Jacod (2009b) and the asymptotic 'variance' is estimated by $c \hat{I}_{1}^{n}$ for suitable constants $c>0$ as specified above. 


\subsection{Results from the simulation study}

In order to assess the finite sample performance of the various centered and standardised realised measures in the simulation studies, we have computed their biases (which should be 0), their standard deviations (which should be 1) and the empirical rejection rates for the levels $10 \%$ and $5 \%$, which are given in the tables in the Appendix. Furthermore, we have studied quantile-quantile plots and density plots of the distributions of the various realised measures, which are not presented here due to space constraints.

\subsubsection{Preceding results regarding the spot volatility estimation}

Before we evaluate the results from the simulation study for the various realised measures, we briefly focus on spot volatility estimation first since spot volatility estimators are used in the estimator of the jump part of the asymptotic 'variance' of the realised variance.

The are various methods for estimating the squared spot volatility, i.e. $\sigma^{2}$, see e.g. Aitt-Sahalia \& Jacod (2009b), Bandi \& Renò (2010), Foster \& Nelson (1996), Kristensen (2010), Lee \& Mykland (2008).
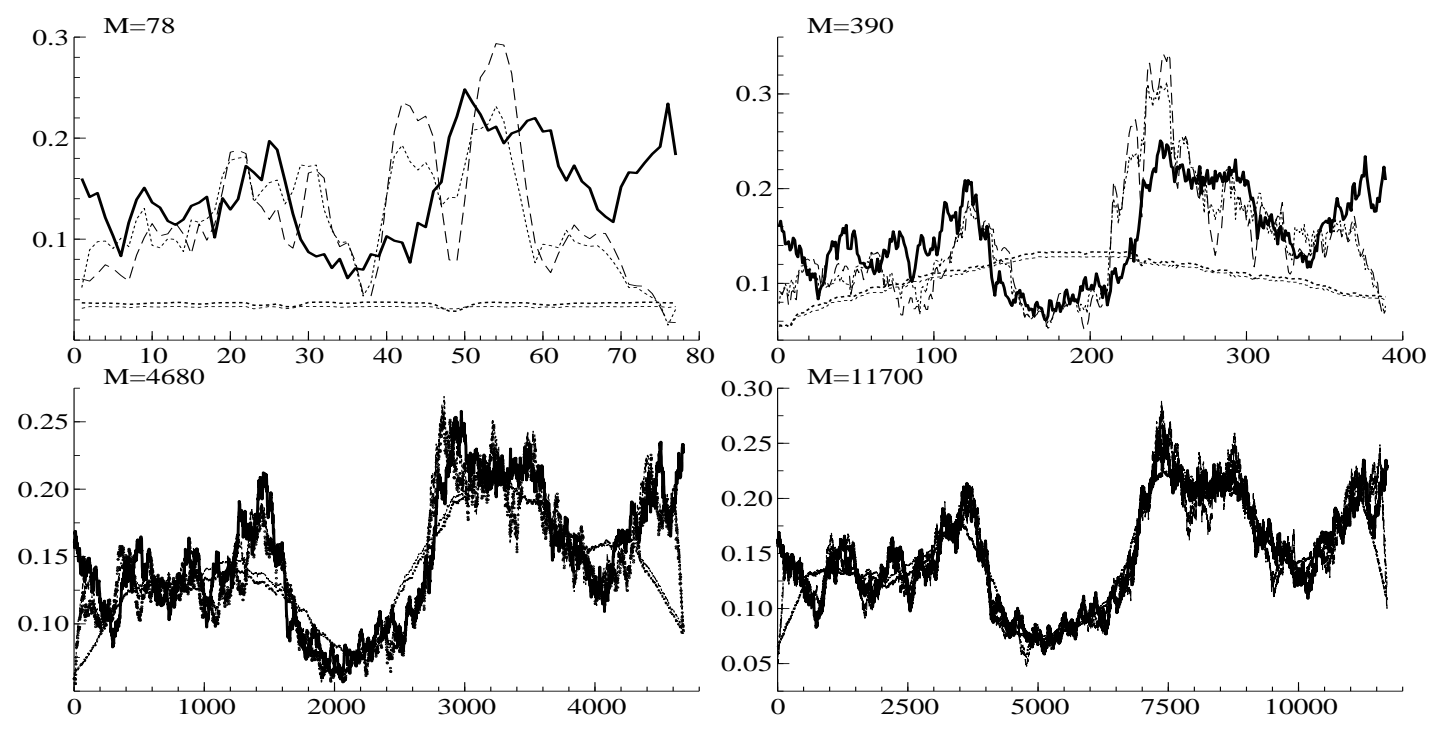

Figure 1: Comparison of various spot volatility estimates for the square-root diffusion model for different numbers of intra-daily observations $M$.

Here we focus on two approaches: We use locally averaged truncated realised variance and locally averaged realised bipower variation as described above. We compute both estimators for two different choices of the local window size $k_{n}$. We work with $\Delta_{n}=1 / n$ and $k_{n} \in\left\{\left\lfloor\Delta_{n}^{-1 / 2}\right\rfloor,\left\lfloor 50 \Delta_{n}^{-1 / 4}\right\rfloor\right\}$. Note that both choices satisfy $k_{n} \rightarrow \infty, k_{n} \Delta_{n} \rightarrow 0$. Clearly, $\left\lfloor\Delta_{n}^{-1 / 2}\right\rfloor \leq\left\lfloor 50 \Delta_{n}^{-1 / 4}\right\rfloor$ for our choices of $\Delta_{n}$. Note that Aït-Sahalia \& Jacod (2009b) used the bigger window size whereas Lee \& Mykland (2008) suggested to use a relatively small window to ensure that the computing load is not too high. Throughout the various scenarios in the simulation study, we always obtained significantly better results for the smaller window $k_{n}=\left\lfloor\Delta_{n}^{-1 / 2}\right\rfloor$, using the root mean square error (RMSE) to compare the various spot estimators. The bigger window size resulted in over-smoothing, which we can clearly 

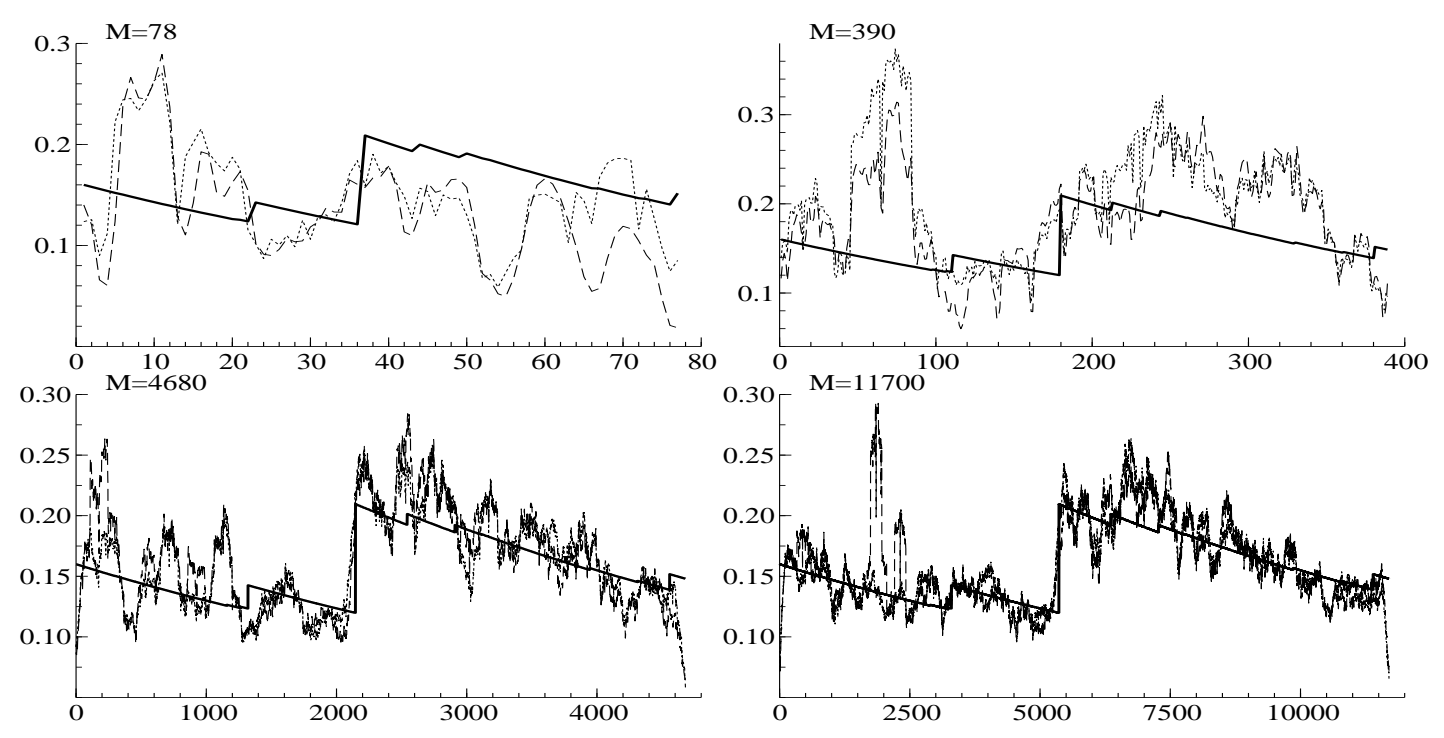

Figure 2: Comparison of various spot volatility estimates for the BNS model, where $\sigma^{2}$ is a nonGaussian Ornstein Uhlenbeck process with inverse Gaussian innovations for different numbers of intra-daily observations $M$.

see in Figure 1 and Figure 2. Figure 1 presents a plot of the simulated $\sigma^{2}$ following a square-root diffusion process (represented by the thick solid line). The dashed and dotted lines represent the locally averaged TRV and R2V, where the smoother lines come from the bigger window size. Figure 2 compares the various spot volatility estimators when $\sigma^{2}$ is sampled from a non-Gaussian Ornstein Uhlenbeck process with inverse Gaussian innovations. Note that $M$ denotes the number of intra-daily observations. I.e. the numbers $M=78,390,4680,11700$ correspond to 5 minute, 1 minute, 5 second and 2 second observations in an equity market which is open 6.5 hours a day. We clearly observe that the spot volatility estimates are rather noisy measures of the underlying volatility. With increasing frequency of the data, however, their performance improves.

Note that we used the simulation design from Aitt-Sahalia \& Jacod (2009b) for the Browniandriven stochastic volatility process and, hence, we used their threshold choice for computing TRV. When comparing the performance of the locally averaged TRV and R2V for estimating the spot volatility based on the RMSE, we obtained very similar results throughout the simulation study. But we can nevertheless formulate some tendencies:

- TRV tends to perform better than R2V in the absence of jumps in the price;

- In the presence of (smaller) jumps in the price, R2V tends to perform better for lower frequencies (up to $\mathrm{M}=390$ ), whereas TRV tends to perform better for higher frequencies;

- In the BNS jump-driven stochastic volatility model, R2V tends to perform better.

- In the presence of rather big jumps in the price, TRV tends to perform better.

Clearly, the performance of TRV can be improved by using a data driven, dynamic threshold, see e.g. Aït-Sahalia \& Jacod (2010), Corsi et al. (2010), Mancini \& Gobbi (2010). However, given that the differences between the two estimators were not that big and if they were, than R2V was in 
most cases the better estimator, from a practical point of view, R2V seems to be the winner in that direct comparison given that it does not require to choose a threshold and leads nevertheless to fairly reasonable estimates. Furthermore, we conclude that the choice of the window size $k_{n}$ seems to be more important than the particular choice of the realised measure. In our simulation experiment, $k_{n}=\left\lfloor\Delta_{n}^{-1 / 2}\right\rfloor$ did a fairly good job for the various models studied here.

\subsubsection{Results for realised variance}

How good is the finite sample performance of realised variance in the presence of jumps? In order to answer that question we have computed the difference of realised variance and the daily quadratic variation of the simulated $\log$-price process and have scaled it suitably by various estimators for the asymptotic 'variance' (and divided it by $\sqrt{\Delta_{n}}$ ), which asymptotically should be standard normally distributed. The various statistics are denoted by RV, RV2, pRV, pRV2 and cRV as defined above and the Tables in the Appendix present the bias, the standard deviation and the empirical rejection rate at $10 \%$ and $5 \%$ level for various numbers $M=\left\lfloor 1 / \Delta_{n}\right\rfloor$ of intra-daily observations.

Our general findings are as follows: We obtain good finite sample results (in terms of the bias being close to 0 , the standard deviation being close to 1 and the empirical rejection rates being close to the theoretical level of $10 \%$ and 5\% respectively, for $M$ between 78 and 195 and higher, corresponding to 2-5 min and more frequent returns in an equity market. In general, the results for the log-transformed realised variance were better throughout the simulation study, but we do not report them here and rather focus on the slightly worse results for the raw realised variance since this quantity is more widely used in practice. Note in particular, that the finite sample bias only seems to go away when one studies data at higher frequencies than 1 minute data in a 6.5 hour market, whereas the bias is noticeably smaller for $\log$-transformed realised variances.

Note that throughout the simulation study, we have chosen the model parameters such that the expected integrated variance stays the same. Further, the jump part of the quadratic variation compared to the total variation amounts to $15 \%$. We have rerun the simulations also for the percentage of the jump part being $30 \%$, but got very similar results as before. Our main findings are as follows:

- The finite sample performance becomes good from 5 minute data onwards to more frequent data;

- The finite sample bias goes to zero for data sampled at 1 minute intervals and more frequent data;

- The activity of the jumps in the price process does not seem to have a big impact on the finite sample performance of realised variance, see the results from model IAP, i.e. Tables 7, 8, 9. In fact, it seems like the finite sample performance gets even a bit better for jumps of higher activity.

- In the presence of jumps, realised variance has to be scaled by one of the new estimators derived in this paper and in Veraart (2010) which account for jumps, otherwise the finite sample performance is not good and gets clearly worse when the frequency of the data increases, see the performance of the statistics cRV in the Tables for more details, where the centered realised variance is only standardised by an estimator of the continuous part of the asymptotic 'variance'.

- When comparing the performance of the various standardised realised variances, we observe that $\mathrm{pRV}$ tends to perform best for low frequencies (up to $\mathrm{M}=390$ ), followed closely by pRV2. 
However, for higher frequencies, using the new estimator $\widehat{D}^{(1), n}$ leads to better finite sample results, see RV and RV2 in the Tables in the Appendix.

- In the presence of common jumps of the volatility and the price process (model BNSCJ), we still observe a good finite sample performance although the asymptotic theory does not hold in that situation anymore. We have carried out that experiment for different choices of $\rho$, which scales the jumps in the volatility to a different level for the price jumps. Note that for $\rho=-0.2$, the jump part of the quadratic variation amounts to approximately $4.7 \%$ of the total quadratic variation, whereas $\rho=-0.4$ corresponds to $16.7 \%$ with our choice of parameters, see Tables 18, 19 and Figure 5.

Note that as before we expect that the performance of the threshold based estimators for the jump part of the asymptotic 'variance' can be improved when choosing a better threshold as discussed in the previous section. However, from an applied point of view, it is encouraging to see that pRV has such a good finite sample performance, where the jump part of the asymptotic 'variance' is estimated based on the difference of generalised realised variance and realised multipower variation and the spot volatility is estimated based on the threshold concept, where a rather crude threshold has been used.

\subsubsection{Results for (truncated) realised (multi-) power variation}

Besides the question of drawing inference on the entire quadratic variation, it is also of interest to study the continuous and the jump part of the quadratic variation separately. We have computed various jump-robust realised measures denoted by TRV, TR2V, R2V, R3V, R4V as defined above and have analysed their finite sample performance in the same simulation studies as realised variance.

Remark Note that we have included realised bipower variation, $\mathrm{R} 2 \mathrm{~V}$, in the simulation experiment even in the presence of jumps although its asymptotic distribution is not Gaussian in that case, see Vetter (2010) for the detailed results. However, truncated realised bipower variation, TR2V, has the corresponding Gaussian limit and, hence, we keep the R2V results to see more clearly the impact of the truncation. So it should be stressed that we cannot expect to get the expected results of standard deviation being equal to 1 and empirical rejection rates being close to $10 \%$ and $5 \%$ for $\mathrm{R} 2 \mathrm{~V}$ as soon as there are jumps in the price present. However, we would still expect to find a bias tending to zero as $\Delta_{n} \rightarrow 0$. Further, note that the multipower results only hold theoretically as long as assumption (A) is satisfied.

The main findings for the jump-robust realised measures are as follows. In the absence of jumps in the price, the performance of the various jump-robust measures is very similar and generally good, but we observe that the finite sample bias at low frequencies tend to be fairly big for R3V and R4V see e.g. Table 3 and 14.

In the presence of jumps in the price process, the general trend we observe is as follows:

- If there are rare jumps, the jump robust measures perform fairly well.

- However, if there are (finitely) many small jumps, our estimation results clearly show a (positive) finite sample estimation bias for all jump robust measures. Note here that the sign of the bias for the truncation based methods could potentially change with a different choice of the threshold. 
- If the price process consists of a Brownian semimartingale and a highly active jump process (e.g. a tempered stable Lévy process), the jump-robust measures show signs of failure. In particular, the more active the jump process gets, the worse results we obtain, see Tables 7,8 , 9.

- Altogether we observe that, in the presence of many jumps, R4V tends to perform best out of the various jump-robust measures studied here at lower frequencies (up to 1 minute data).

- In the BNS modelling framework (BNSCJ), we also obtain reasonable finite sample results for $\mathrm{R} 2 \mathrm{~V}$, which is somewhat surprising given that the asymptotic theory does not hold here. That might be due to the fact that the jump activity of the inverse Gaussian subordinator is still rather low.

\subsection{Concluding remarks regarding the simulation study}

In general, we have observed that the feasible asymptotic theory for realised variance seems to hold also in finite samples when data at a frequency of 2-5 minutes or higher are studied. The differences between the various estimators of the asymptotic 'variance' of realised variance are not that big, but generally, we obtained the best results for $\mathrm{pRV}$ at lower frequencies.

When using jump-robust realised measures using the truncation principle, the multipower concept or a combination of both, we found that they perform quite well in the presence of rare jumps. However, as soon as there are many small jumps and in particular, if there is a very active jump process present, then the finite sample performance of the jump-robust measures is not good any longer. This is in line with the findings of Barndorff-Nielsen et al. (2005) and Veraart (2010). In order to get somewhat reliable measures in that situation, higher order multipower variations such as R4V (possibly combined with a dynamically chosen threshold) seem to be the best choice. Further, using $\log$-transformed versions of realised variance rather than the raw realised variance also improves the finite sample performance.

While inference on the entire quadratic variation of the log-price process based on realised variance using the new estimator of the asymptotic 'variance' of RV presented in this paper and the one presented in Veraart (2010) turns out to work well even in finite samples and also in the presence of very active jump processes, the finite sample results for drawing inference on the continuous part or the jump part only are generally not that good when many jumps are present.

\section{Empirical study of SPY data}

After we have checked the finite sample performance of various feasible estimators of the quadratic variation of an Itô semimartingale, we use our findings now to carry out an empirical study of some high frequency equity data.

\subsection{Data description}

We use data from the Standard \& Poor's Depository Receipt (SPY), which is a very liquid, exchangetraded fund which holds all of the S\&P 500 Index stocks. The sample we work with covers a period from 3 August 1998 to 31 July 2008, i.e. 10 years of data. We focus on mid-quote data constructed based on the quote data taken from the TAQ database through the Wharton Research Data Services (WRDS) system. The SPY data were recorded at the AMEX from 1998-2002 and at the PACIF from 

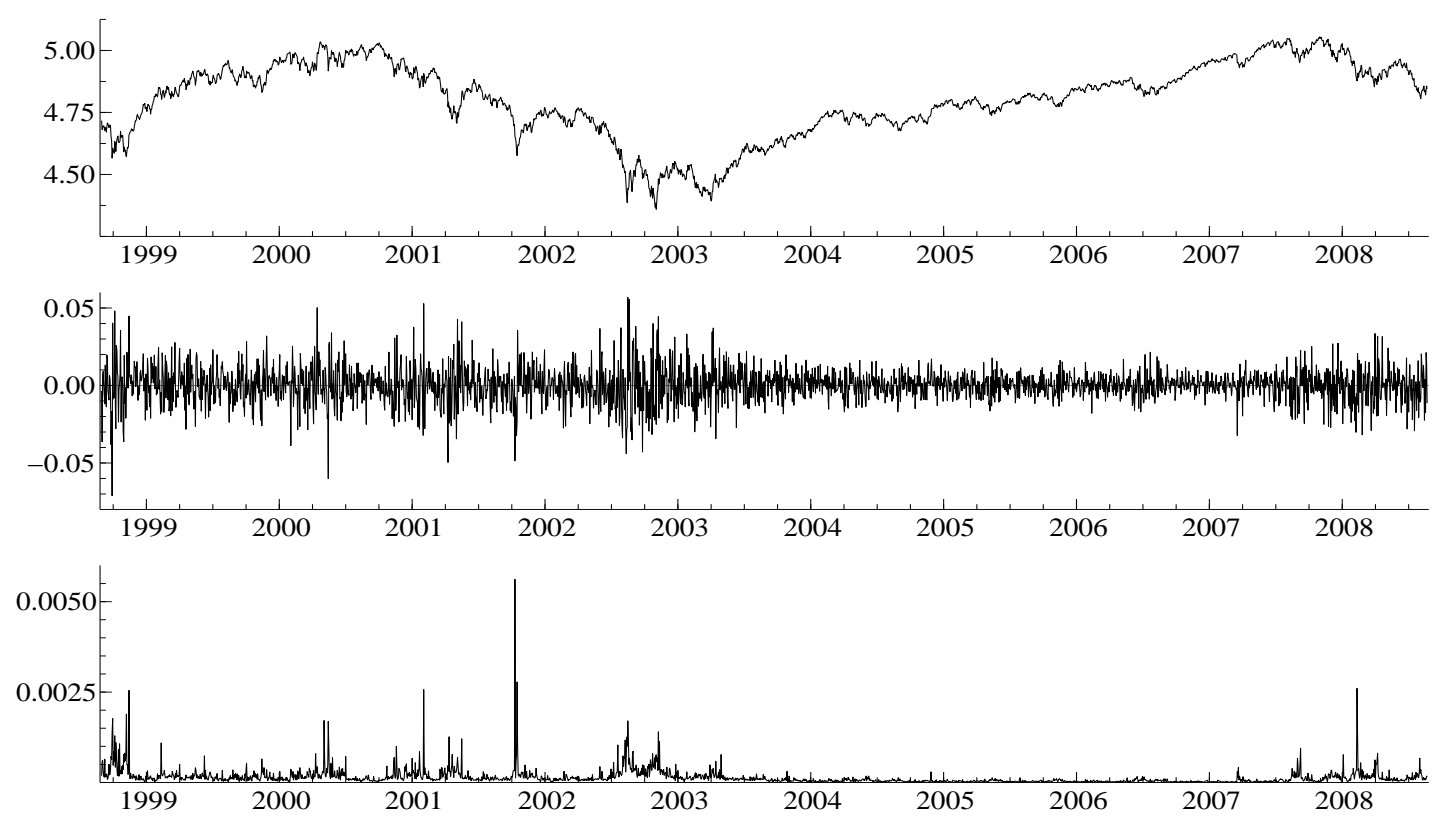

Figure 3: SPY data from 3 August 1998 to 31 July 2008: (a) Cleaned daily log-mid-prices; (b) daily $\log$-mid-price returns; (c) time series of daily realised variances based on five minute returns.

2003-2008. The high frequency data have been kindly supplied to the author by Asger Lunde and have been cleaned using the methods described in Barndorff-Nielsen et al. (2008b). We have sampled the data at five minute intervals (using the previous tick method) and have obtained $n=2515$ days of 78 observations each, i.e. 196,170 data points. A plot of the cleaned SPY $\log$-price data, the returns and the time series of the realised variances is given in Figure 3.

The aim of this study is to analyse the impact of jumps on inference for realised variance. In order to do that, we identify days which have a significant jump contribution and then check how much wider the confidence intervals for realised variance become on those days.

\subsection{Testing for jumps and inference on quadratic variation in the presence of jumps}

As already mentioned above, there is a wide literature on testing for jumps using high frequency data. In our empirical study, we follow Huang \& Tauchen (2005) who found out in detailed Monte Carlo studies that the ratio statistic of the Barndorff-Nielsen \& Shephard (2006) test using a maximum adjustment in the denominator has the best size property out of the various test statistics studied, including the properly scaled difference of realised variance and realised bipower (often referred to as raw test statistic), the difference of the log-transformed realised variance and realised bipower and the ratio of the difference of realised variance and realised bipower divided by realised variance. When carrying out the test, we get the following results, as reported in Table 2.

Note that Huang \& Tauchen (2005) did not find significant differences when the test statistics were scaled by realised tripower or realised quadpower variation, respectively. This is basically in line with what we find here. In our empirical study, we reject the null hypothesis slightly more often when using realised quadpower variation as the scaling factor, which is in line with what we expect: Since realised quadpower variation averages over more returns than realised tripower variation, we 
Table 2: Empirical results from the maximum adjusted BNS ratio test.

\begin{tabular}{cccc}
\hline Significance level & M & $\begin{array}{c}\text { Number and percentage } \\
\text { of rejections }\end{array}$ & $\begin{array}{c}\text { Average contribution of the } \\
\text { jump part of the CIs }\end{array}$ \\
\hline $99 \%$ & 39 & $632(25.12 \%)$ & $55.61 \%$ \\
& 78 & $931(37.01 \%)$ & $64.96 \%$ \\
& 130 & $1136(45.16 \%)$ & $58.82 \%$ \\
& 195 & $1328(52.80 \%)$ & $53.75 \%$ \\
& 390 & $1779(70.73 \%)$ & $40.52 \%$ \\
\hline $99.90 \%$ & 39 & $390(15.50 \%)$ & $57.07 \%$ \\
& 78 & $658(26.16 \%)$ & $67.43 \%$ \\
& 130 & $844(33.55 \%)$ & $60.59 \%$ \\
& 195 & $1052(41.82 \%)$ & $54.63 \%$ \\
& 390 & $1520(60.43 \%)$ & $41.38 \%$ \\
\hline
\end{tabular}

expect it to be generally smaller in the presence of jumps, which results in more rejection of the null hypothesis given that the scaling factor appears in the denominator of the ratio statistic.

Our test signals the presence of a significant jump contribution to the total quadratic variation (at 99\% significance level) on 25-70\% of the days - depending on which data frequency we use for computing the test statistic. In the following we will just refer to those days as jump days. Furthermore, we observe that the number of rejections of the null hypothesis increases when the number of intra-daily observations, denoted by $M$, increases. That is also in line with Huang \& Tauchen (2005) findings that when data at lower frequencies are considered, jumps tend to average out and are not necessarily detected by the jump test anymore.

However, in general we can say that the results from the jump test are rather surprising. Rejecting the null hypothesis that frequently is not in line with earlier empirical studies in the literature, see e.g. Huang \& Tauchen (2005). However, there are also more recent studies, see e.g. Aït-Sahalia \& Jacod (2009a) whose findings point towards the presence of a very active jump component even for highly liquid stocks. If this is true, then we would expect to reject the null hypothesis of no jumps very often and ideally on (almost) all days.

After we have tested for each day in the sample whether there was a significant jump part in the quadratic variation and hence, have identified those jump days, we study how the jumps affect the size of the confidence bounds for realised variance on these jump days. In order to do that, we have computed the average length of the confidence bounds assuming that there were no jumps (i.e. estimating the asymptotic 'variance' by $2 \hat{I}$ ) and the average length of the confidence bounds which accounts for jumps (i.e. estimating the asymptotic 'variance' by $2 \hat{I}+2 \hat{D}$ ). We find that the part of the confidence bounds due to the presence of jumps amounts to approximately $40-60 \%$ of the total length, see the last column in Table 2. Further, we observe that the average jump contribution to the total length of the CIs tends to decrease with increasing number of intra-daily observations.

In order to get an additional visual impression on how the confidence bounds for realised variance change in the presence of jumps, we provide a plot of the confidence bounds for RV which account for jumps and add the ones which ignore jumps. In Figure 4, we plot the time series of realised variances with the corresponding 95\% confidence bounds for the days when the maximum adjusted BNS ratio test based on ten minute data using realised tripower for the scaling rejects the null hypothesis at the 99.9\% level, which was the case on 390 days. The crosses indicated the realised variances, the bars 


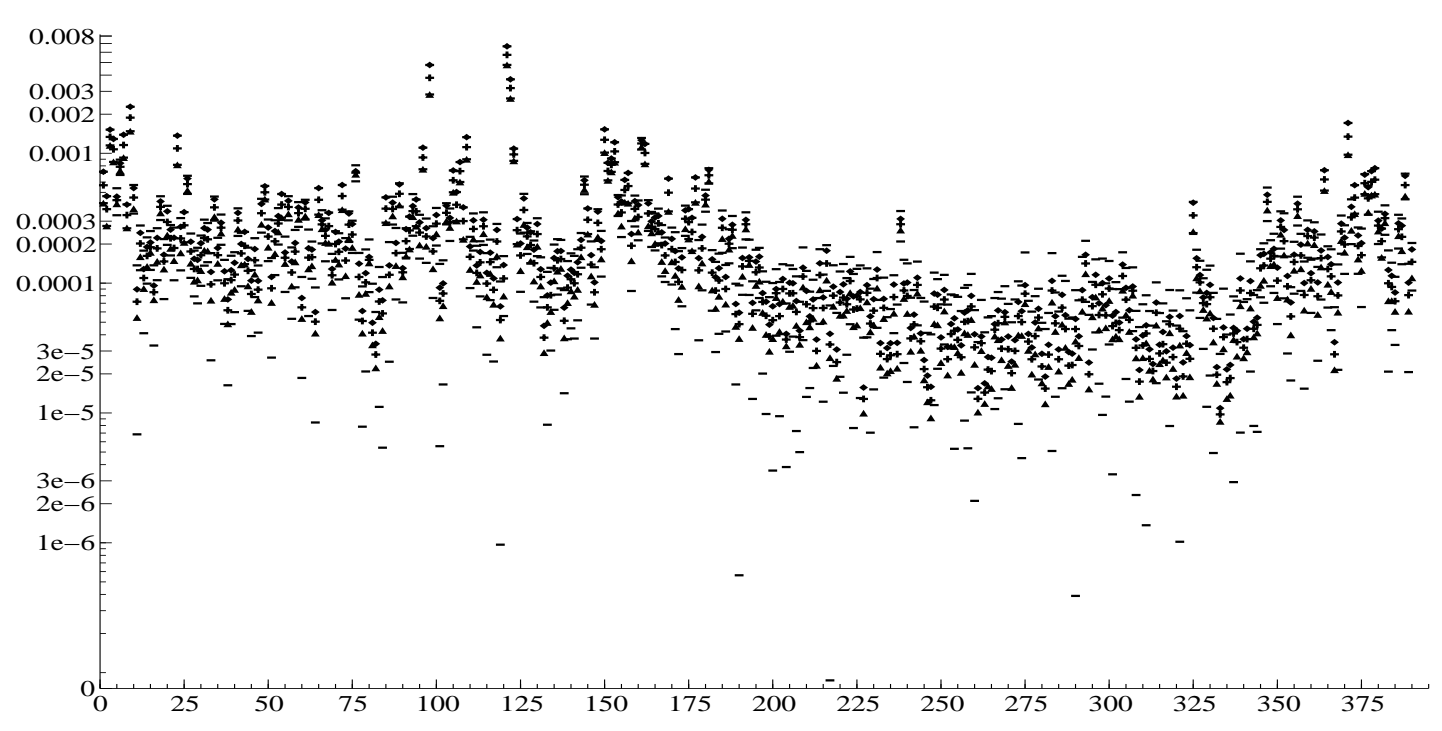

Figure 4: Here we plot the time series of realised variances (using the $\log$-scale) with the corresponding 95\% confidence bounds for the days when the maximum adjusted BNS ratio test based on ten minute data using realised tripower for the scaling rejects the null hypothesis at the $99.9 \%$ level, which was the case on 390 days.

denote the confidence bounds which account for jumps and the triangles/circles denote the confidence bounds which ignore jumps. We see that the confidence intervals which account for jumps are the wider ones, which is what we expect.

\section{Conclusion}

This paper has investigated how accurate the asymptotic distribution of realised variation measures is in finite samples when there are finite or infinite activity jumps in the underlying price process. In order to do that, we have proposed a new estimator for the jump part of the asymptotic 'variance' of centered realised variance. Then, we have carried out a detailed Monte Carlo study, where we have compared the finite sample performance of 20 different centered and standardised realised measures. Here we were in particular interested in the performance of realised variance in the presence of jumps. To the best of our knowledge, this is the first paper, which has addressed this important question given the strong evidence for the presence of jumps in financial data, see e.g. Barndorff-Nielsen \& Shephard (2004b, 2006), Huang \& Tauchen (2005), Lee \& Mykland (2008), Aït-Sahalia \& Jacod $(2009 b)$. We have studied the finite sample performance of the newly established asymptotic theory for realised variance, see Jacod (2008), Veraart (2010). In our simulation experiment we have compared the results, when the asymptotic 'variance' is estimated based on two different spot volatility estimators: locally averaged realised bipower variation and locally averaged truncated realised variance. In general, we have obtained good results in the Monte Carlo experiments, where the results for the log-transformed realised variance were always slightly better than for the realised variance. Furthermore, both estimators for the jump part of the realised variance provide fairly similar results. The only noticeable differences between the two spot volatility estimators were that truncated realised variance seems to perform slightly better in the stochastic volatility jump diffusion model, whereas 
realised bipower variation seems to be slightly better in models which allow for jumps in the volatility process.

Regarding the size of the local window $k_{n}$, we have obtained good results when choosing $k_{n}=$ $1 / \sqrt{\Delta_{n}}$ which furthermore is not that computationally intense. Also, we have clearly seen that the window size $k_{n}$ has a great impact on the performance of the spot volatility estimator, whereas the particular choice of the spot volatility estimator, i.e. which jump-robust realised quantity is used, does not seem to matter much.

Furthermore, Monte Carlo results suggest that our feasible limit theory might still work even in the case of common jumps of the volatility and the price process as long as the common jump part is rather small.

Regarding the so-called jump robust measures such as realised multipower variation and truncated realised measures, we found that the finite sample performance was not as good as expected. While we have obtained fairly good results for rare jumps in the price, we could clearly see that the performance of the jump robust realised measures studied here worsens dramatically when the jump activity increases.

In addition to the simulation experiment, we have applied the feasible asymptotic theory for realised variance to high frequency SPY data and have computed the confidence intervals for realised variance on the days which were identified as jump days using the maximum adjusted BanrdorffNielsen \& Shephard ratio test. We have clearly seen that the confidence bounds of realised variance which account for jumps are significantly wider than the ones suggested by Barndorff-Nielsen \& Shephard (2002) in the absence of jumps.

In our empirical study, we have worked with rather low frequency data to ensure that the data are not affected by market microstructure noise. However, in future research, it will be interesting to study the impact of jumps on inference on the quadratic variation of the price process if the efficient price is contaminated by market microstructure effects and, hence, not directly observed. In particular, ultra high frequency data (e.g. one second returns) are typically prone to such effects and, hence, volatility estimation and inference in such a framework is carried out using noise robust volatility estimators, see e.g. Zhang et al. (2005), Hansen \& Lunde (2006), Zhang (2006), Barndorff-Nielsen \& Shephard (2007), Barndorff-Nielsen et al. (2008a), and Podolskij \& Vetter (2009). Volatility estimators which are robust to market microstructure effects have a slower rate of convergence and do not achieve the square root speed of convergence of realised variance and realised multipower variation. Hence, it will be interesting to study in simulation studies and also empirically which estimators perform better in finite samples in the presence of jumps and market microstructure effects.

\section{A Technical assumptions}

\section{A.1 Assumptions on $X$ and $\sigma$}

For the central limit theorems of realised variance and realised multipower variation we need some regularity assumptions and a structural assumption for the volatility process which we introduce in the following. All the technical assumptions are taken from Jacod (2008, p. 5-6) and Aitt-Sahalia \& Jacod (2009b, p. 187,204).

We work with a probability space denoted by $\left(\Omega, \mathcal{A},\left(\mathcal{F}_{t}\right)_{t \geq 0}, \mathbb{P}\right.$ ) (or with a slightly enlarged version of the original probability space). Let

$$
\phi_{r}(x)= \begin{cases}1 \wedge|x|^{r}, & \text { if } 0<r<\infty \\ \mathbb{I}_{\mathbb{R} \backslash\{0\}}(x), & \text { if } r=0 .\end{cases}
$$


We focus on Itô semimartingales $X$ which satisfy the following conditions.

Assumption (H): $X$ is an Itô semimartingale as defined in (1) and (2) and the processes $\left(b_{t}\right)_{t \geq 0}$ and $\left(\int \phi_{2}(x) F_{t}(d x)\right)_{t \geq 0}$ are locally predictable. Note that $F_{t}(\omega, d x)$ is the image of the Lebesgue measure on $\mathbb{R}$ by the predictable map $x \mapsto \delta(\omega, t, x)$. Further, the process $\left(\sigma_{t}\right)_{t \geq 0}$ is càdlàg adapted.

Assumption (L-s): (With $s \in[0,2])$. $X$ is an Itô semimartingale as defined above satisfying assumption $(\mathrm{H})$ and the volatility process $\sigma$ has the form

$$
\sigma_{t}=\sigma_{0}+\int_{0}^{t} \widetilde{b}_{u} d u+\int_{0}^{t} \widetilde{\sigma}_{u} d W_{u}+\int_{0}^{t} \widetilde{\sigma}_{u}^{\prime} d W_{u}^{\prime}+\kappa(\tilde{\delta}) \star(\underline{\mu}-\underline{\nu})_{t}+\kappa^{\prime}(\tilde{\delta}) \star \underline{\mu}_{t},
$$

and

- $W^{\prime}$ is another Brownian motion on the space $\left(\Omega, \mathcal{A},\left(\mathcal{F}_{t}\right)_{t \geq 0}, \mathbb{P}\right)$, which is independent of $W$;

- the process $\left(\widetilde{b}_{t}\right)_{t \geq 0}$ is optional and locally bounded;

- the processes $\left(b_{t}\right)_{t \geq 0},\left(\widetilde{\sigma}_{t}\right)_{t \geq 0},\left(\widetilde{\sigma}_{t}^{\prime}\right)_{t \geq 0}$ are adapted left-continuous with right limits in $t$, and locally bounded;

- the functions $\delta, \tilde{\delta}: \Omega \times \mathbb{R}_{+} \times \mathbb{R} \rightarrow \mathbb{R}$ are defined such that $F_{t}(\omega, d x)$ is the image of the Lebesgue measure on $\mathbb{R}$ by the map $x \rightarrow \delta(\omega, t, x) ; \delta(\omega, t, x)$ and $\tilde{\delta}(\omega, t, x)$ are predictable, left-continuous with right limits in $t$. Further $|\delta(\omega, t, x)| \leq \gamma_{k}(x)$ and $|\delta(\omega, t, x)| \leq \tilde{\gamma}_{k}(x)$ for all $t \leq T_{k}(\omega)$ with deterministic functions $\gamma_{k}$ and $\hat{\gamma}_{k}$ on $\mathbb{R}$ with $\int \phi_{s} \circ \gamma_{k}(x) d x<\infty$ and $\int \phi_{2} \circ \tilde{\gamma}_{k}(x) d x<\infty$ and $\left(T_{k}\right)_{k \geq 1}$ is a sequence of stopping times increasing to $+\infty$.

Note that assumption (L-s) basically says that the stochastic volatility process $\sigma$ has to be an Itô semimartingale, too. Further, the parameter $s$ can be regarded as an generalised Blumenthal Getoor index, see Blumenthal \& Getoor (1961), measuring the jump activity of the jump part part of $X$. See Jacod (2008, Remark 2.1) for a wide class of processes which in fact satisfy the above assumptions.

Assumption(H'): Assumption (H) holds and $\sigma_{t}^{2}>0$ and $\sigma_{t-}^{2}>0$ for all $t$ a.s..

Assumption (G1): Assumptions (L-s) and (H') hold (for some $s \in[0,2])$ and further

1. All paths $t \mapsto \sup _{x \in E} \frac{|\delta(\omega, t, x)|}{\gamma(x)}$ and $t \mapsto \sup _{x \in E} \frac{|\tilde{\delta}(\omega, t, x)|}{\gamma(x)}$ are locally bounded, where $\gamma$ is a (nonrandom) nonnegative function satisfying $\left.\int_{E}(\gamma(x))^{2} \wedge 1\right) \lambda(d x)<\infty$.

2. All paths $t \mapsto \delta_{t}^{\prime}(\omega)$ are left-continuous with right limits on the semiopen set $[0, \tau(\omega))$. Here $\delta_{t}^{\prime}(\omega)=\int \kappa \circ \delta(\omega, t, x) \lambda(d x)$, if the integral is well-defined, otherwise we set $\delta_{t}^{\prime}(\omega)=\infty$.

Assumption (G2): Assumption (G1) holds and $\left|b_{t}\right|+\left|\sigma_{t}\right|+\left|\tilde{b}_{t}\right|+\left|\tilde{\sigma}_{t}\right|+\left|\tilde{\sigma}_{t}^{\prime}\right| \leq K$, and $|\delta(t, x)| \leq \gamma(x)$, $|\tilde{\delta}(t, x)| \leq \gamma(x)$ and also $\gamma(x) \leq K$ for some constant $K$.

Assumption (A): Assumption (L-s) holds for $s<1$, (H') is satisfied and $\frac{s}{2-s}<\mathbf{r}_{-}<\mathbf{r}_{+}<1$.

Assumption (B): Assumption (L-s) holds and $s \leq \frac{4 \omega-1}{2 \omega}$ (hence $\omega \geq 1 / 4$ and $s<1$ ).

Assumption (C): Assumption (L-s) holds for $s<1$, (H') is satisfied and $I \in \mathbb{N}$ with2 $<I<$ $\frac{2}{s}(2-s)$. Further, $X$ and $\sigma$ do not have common jumps. 


\section{B Proofs}

\section{B.1 Notation and preliminary remarks}

Throughout the proofs we use the following notation:

$$
\delta_{i}^{n}=\sigma_{(i-1) \Delta_{n}} \Delta_{i}^{n} W, \quad \theta_{i}^{n}=\left|\Delta_{i}^{n} X\right| \mathbb{I}_{\left\{\left|\Delta_{i}^{n} X\right| \leq \alpha \Delta_{n}^{\omega}\right\}},
$$

for $\alpha>0$ and $\omega \in\left(0, \frac{1}{2}\right)$. Further, we write $\mathbb{E}_{i-1}^{n}(\cdot)=\mathbb{E}\left(\cdot \mid \mathcal{F}_{(i-1) \Delta_{n}}\right)$ for the conditional expectation.

Furthermore, we write $X=X^{\prime}+X^{d}$, where $X^{c}$ denotes the continuous part and $X^{d}$ the discontinuous part of $X$ and

$$
\begin{aligned}
X_{t}^{\prime} & =X_{0}+\int_{0}^{t} b_{s} d s+X_{t}^{c}, \quad X_{t}^{c}=\int_{0}^{t} \sigma_{s} d W_{s}, \\
X^{d} & =\int_{0}^{t} \int_{E} \kappa(\delta(s, x)) \star(\mu-\nu)(d s, d x)+\int_{0}^{t} \int_{E} \kappa^{\prime}(\delta(s, x)) \star \mu(d s, d x) .
\end{aligned}
$$

In the following, we will apply the following inequalities, which are valid under Assumption (G2), see e.g. Aït-Sahalia \& Jacod (2009b), Jacod (2008), Vetter (2010), where $K_{i}>0$ for $i \in \mathbb{N}$ denote positive constants:

$$
\begin{array}{lll}
\mathbb{E}_{i-1}^{n}\left(\left|\Delta_{i}^{n} X^{c}\right|\right) \leq K_{1} \Delta_{n}^{1 / 2}, \quad & \mathbb{E}_{i-1}^{n}\left(\left|\Delta_{i}^{n} X^{\prime}\right|\right) \leq K_{2} \Delta_{n}^{1 / 2}, \quad \mathbb{E}_{i-1}^{n}\left(\left|\Delta_{i}^{n} X^{d}\right|\right) \leq K_{3} \Delta_{n}, \\
& \mathbb{E}_{i-1}^{n}\left(\left|\Delta_{i}^{n} X\right|\right) \leq K_{4} \Delta_{n}^{1 / 2} .
\end{array}
$$

Note that in the following, we will denote by $K>0$ a constant which can change from line to line throughout the proof.

Remark We will prove Proposition 3.1 under the stronger assumption (G2). Using a localisation procedure, it follows from standard arguments, see Jacod (2008) for details, that our result also holds under the weaker assumption (G1).

\section{B.2 Proof}

Proof of Proposition 3.1 The proof of the Proposition is a direct consequence of the previous remark and the following two lemmas.

Lemma B.1 Under Assumption (G2) and if

$$
\widehat{D}_{t}^{\prime n}=\frac{1}{k_{n} \Delta_{n}} \sum_{i=1}^{\left\lfloor t / \Delta_{n}\right\rfloor}\left(\Delta_{i}^{n} X\right)^{2} \mathbb{I}_{\left\{\left|\Delta_{i}^{n} X\right|>\alpha \Delta_{n}^{\omega}\right\}} \sum_{j \in I_{n, t}(i)}\left(\delta_{j}^{n}\right)^{2} \stackrel{\mathbb{P}}{\rightarrow} D_{t}
$$

then $\widehat{D}_{t}^{n} \stackrel{\mathbb{P}}{\rightarrow} D_{t}$, where

$$
\widehat{D}_{t}^{n}=\frac{1}{k_{n} \Delta_{n}} \sum_{i=1}^{\left\lfloor t / \Delta_{n}\right\rfloor}\left(\Delta_{i}^{n} X\right)^{2} \mathbb{I}_{\left\{\left|\Delta_{i}^{n} X\right|>\alpha \Delta_{n}^{\omega}\right\}} \sum_{j \in I_{n, t}(i)}\left(\Delta_{j}^{n} X\right)^{2} \mathbb{I}_{\left\{\left|\Delta_{j}^{n} X\right| \leq \alpha \Delta_{n}^{\omega}\right\}} .
$$


Proof The proof goes along the lines of Ait-Sahalia \& Jacod (2009b, Lemma 1). In particular, we can immediately deduce, see Aït-Sahalia \& Jacod (2009b, equation (67)), that

$$
\frac{1}{\Delta_{n}} \mathbb{E}\left(\left|\left(\theta_{i}^{n}\right)^{2}-\left(\delta_{i}^{n}\right)^{2}\right| \mid \mathcal{F}_{(i-1) \Delta_{n}}\right) \leq \widehat{\Gamma}_{n}
$$

for a sequence $\left(\widehat{\Gamma}_{n}\right)_{n \in \mathbb{N}}$ satisfying $\lim _{n \rightarrow \infty} \widehat{\Gamma}_{n}=0$. Note that the difference $\widehat{D}_{t}^{n}-\widehat{D}_{t}^{\prime n}$ consists of a sum with strictly less than $2 k_{n}\left\lfloor t / \Delta_{n}\right\rfloor$ terms, where each term is smaller than

$$
\frac{1}{k_{n} \Delta_{n}}\left(\Delta_{i}^{n} X\right)^{2} \mathbb{I}_{\left\{\left|\Delta_{i}^{n} X\right|>\alpha \Delta_{n}^{\omega}\right\}}\left|\left(\theta_{j}^{n}\right)^{2}-\left(\delta_{j}^{n}\right)^{2}\right|, \quad \text { for some } i \neq j .
$$

From using the inequalities (16), we get that $\mathbb{E}_{i-1}^{n}\left(\left(\Delta_{i}^{n} X\right)^{2} \mathbb{I}_{\left\{\left|\Delta_{i}^{n} X\right|>\alpha \Delta_{n}^{\omega}\right\}}\right) \leq K \Delta_{n}$. Now, we use two successive conditional expectations and (18) to deduce that each such term is smaller than $\frac{1}{k_{n}} K \widehat{\Gamma}_{n} \Delta_{n}$. So, altogether, we get $\mathbb{E}\left|\widehat{D}_{t}^{n}-\widehat{D}_{t}^{\prime n}\right| \leq K t \widehat{\Gamma}_{n}$ and, hence, the result follows.

Lemma B.2 Under Assumption (G2), equation (17) holds.

Proof In order to prove this result, we adapt the proof given in Aït-Sahalia \& Jacod (2009b, pp. 216218) so that it is suitable for the different result, we prove here.

We introduce a continuous function on $[0,1]$ for any $\rho \in(0,1)$ which is defined by $\psi_{\rho}(x)=$ $\min \left(1,(2-|x| / \rho)^{+}\right)$, and we define two (increasing) processes by

$$
Y(\rho)_{t}^{n}=\frac{1}{k_{n} \Delta_{n}} \sum_{i=1}^{\left\lfloor t / \Delta_{n}\right\rfloor} \psi_{\rho}\left(\Delta_{i}^{n} X\right)\left(\Delta_{i}^{n} X\right)^{2} \mathbb{I}_{\left\{\left|\Delta_{i}^{n} X\right|>\alpha \Delta_{n}^{\omega}\right\}} \sum_{j \in I_{n, t}(i)}\left(\delta_{j}^{n}\right)^{2}, \quad Z(\rho)_{t}^{n}=\widehat{D}_{t}^{\prime n}-Y(\rho)_{t}^{n} .
$$

It is sufficient to prove that we have the following three convergence results for a suitable process $Z(\rho)$ :

$$
\begin{aligned}
\lim _{\rho \rightarrow 0} \limsup _{n} \mathbb{E}\left(Y(\rho)_{t}^{n}\right) & =0, \\
\rho \in(0,1), n \rightarrow \infty & \Rightarrow Z(\rho)_{t}^{n} \stackrel{\mathbb{P}}{\rightarrow} Z(\rho)_{t}, \\
\rho \rightarrow 0 & \Rightarrow Z(\rho)_{t} \stackrel{\mathbb{P}}{\rightarrow} D_{t} .
\end{aligned}
$$

Now we prove (19). Note that $Y(\rho)_{t}^{n}$ is a sum of strictly less than $2 k_{n}\left\lfloor t / \Delta_{n}\right\rfloor$ terms, where each term is given by

$$
\frac{1}{k_{n} \Delta_{n}} \psi_{\rho}\left(\Delta_{i}^{n} X\right)\left(\Delta_{i}^{n} X\right)^{2} \mathbb{I}_{\left\{\left|\Delta_{i}^{n} X\right|>\alpha \Delta_{n}^{\omega}\right\}}\left(\delta_{j}^{n}\right)^{2}, \quad \text { for } i \neq j .
$$

From (16), we get that $\mathbb{E}_{j-1}^{n}\left(\left(\delta_{j}^{n}\right)^{2}\right) \leq K \Delta_{n}$.

Next, we study the term $\psi_{\rho}\left(\Delta_{i}^{n} X\right)\left(\Delta_{i}^{n} X\right)^{2} \mathbb{I}_{\left\{\left|\Delta_{i}^{n} X\right|>\alpha \Delta_{n}^{\omega}\right\}}$. Note that an application of the Markov inequality and the inequalities in (16) leads to $\mathbb{E}_{i-1}^{n}\left(\left(\Delta_{i}^{n} X\right)^{2} \mathbb{I}_{\left\{\left|\Delta_{i}^{n} X\right|>\alpha \Delta_{n}^{\omega}\right\}}\right) \leq K \Delta_{n}^{1+1 / 2-\omega}$. Now we take two successive conditional expectations and get $\mathbb{E}\left(Y(\rho)_{t}^{n}\right) \leq K t \Delta_{n}^{1 / 2-\omega}$. So we can deduce (19). 
Next, we define the process $Z(\rho)$ by

$$
Z(\rho)_{t}=\sum_{q: T_{q}(\rho) \leq t}\left(\Delta X_{T_{q}(\rho)}\right)^{2}\left(1-\psi_{\rho}\left(\Delta X_{T_{p}(\rho)}\right)\right)\left(\sigma_{T_{q}(\rho)-}^{2}+\sigma_{T_{q}(\rho)}^{2}\right),
$$

where, for $q=1,2, \ldots, T_{q}(\rho)$ denote the (successive) jump times of $\mu([0, t] \times\{x: \gamma(x)>\rho / 2\})$. Note that this is the $\gamma$ from Assumption (G1)! From the same arguments as in Aït-Sahalia \& Jacod (2009b), we now obtain (21). Note, in particular, that $1-\psi_{\rho}$ converges to the to the indicator function on $\mathbb{R} \backslash\{0\}$.

Finally, we prove (20). Let $\rho \in(0,1)$ be fixed. For ease of notation, we write $T_{q}=T_{q}(\rho)$. Note that

$$
Z(\rho)_{t}^{n}=\frac{1}{k_{n} \Delta_{n}} \sum_{i=1}^{\left\lfloor t / \Delta_{n}\right\rfloor}\left(1-\psi_{\rho}\left(\Delta_{i}^{n} X\right)\right)\left(\Delta_{i}^{n} X\right)^{2} \mathbb{I}_{\left\{\left|\Delta_{i}^{n} X\right|>\alpha \Delta_{n}^{\omega}\right\}} \sum_{j \in I_{n, t}(i)}\left(\delta_{j}^{n}\right)^{2} .
$$

From the definition of the Poisson process $\mu$, we see immediately that $\left|\Delta X_{s}\right| \leq \rho / 2$ if $s \notin\left\{T_{q}, q=\right.$ $1,2, \ldots\}$.

So, for each $t \geq 0$ and for each $\omega$ and for $n$ large enough, we get: there is no jump time $T_{q}$ in $\left(0, k_{n} \Delta_{n}\right]$ and in $\left(t-\left(k_{n}+1\right) \Delta_{n}, t\right]$. Further, in the interval $\left((i-1) \Delta_{n}, i \Delta_{n}\right]$ (for $\left.i \Delta_{n} \leq t\right)$ there is at most one $T_{q}$. If there is no jump time in the interval, then $\psi_{\rho}\left(\Delta_{i}^{n} X\right)=1$. So, as soon as $n$ is large enough, we obtain that

$$
Z(\rho)_{t}^{n}=\sum_{q: k_{n} \Delta_{n}<T_{q} \leq t-\left(k_{n}+1\right) \Delta_{n}} \zeta_{q}^{n},
$$

where $i(n, q)=\inf \left(i: i \Delta_{n} \geq T_{q}\right), I^{\prime}(n, q)=\left\{j: j \neq i(n, q),|j-i(n, q)| \leq k_{n}\right\}$ and

$$
\zeta_{q}^{n}=\frac{1}{k_{n} \Delta_{n}}\left(\Delta_{i(n, q)}^{n} X\right)^{2} \mathbb{I}_{\left\{\left|\Delta_{i(n, q)}^{n} X\right|>\alpha \Delta_{n}^{\omega}\right\}}\left(1-\psi_{\rho}\left(\Delta_{i(n, q)}^{n} X\right)\right) \sum_{j \in I^{\prime}(n, q)}\left(\delta_{j}^{n}\right)^{2} .
$$

We have to show that for any $q$ :

$$
\zeta_{q}^{n} \stackrel{\mathbb{P}}{\rightarrow}\left(\Delta X_{T_{q}}\right)^{2}\left(1-\psi_{\rho}\left(\Delta X_{T_{q}}\right)\right)\left(\sigma_{T_{q}-}^{2}+\sigma_{T_{q}}^{2}\right) .
$$

From Aït-Sahalia \& Jacod (2009b), we know that

$$
\frac{1}{k_{n} \Delta_{n}} \sum_{j \in I_{-}^{\prime}(n, q)}\left(\delta_{j}^{n}\right)^{2} \stackrel{\mathbb{P}}{\rightarrow} \sigma_{T_{q}-}^{2}, \quad \quad \frac{1}{k_{n} \Delta_{n}} \sum_{j \in I_{+}^{\prime}(n, q)}\left(\delta_{j}^{n}\right)^{2} \stackrel{\mathbb{P}}{\rightarrow} \sigma_{T_{q}}^{2},
$$

where $I_{-}^{\prime}(n, q)$ and $I_{+}^{\prime}(n, q)$ are the subsets of $I^{\prime}(n, q)$ containing all $j$ smaller and bigger, respectively, than $i(n, q)$. Next, from Ait-Sahalia \& Jacod $(2009 b)$ we also know that

$$
\left(\Delta_{i(n, q)}^{n} X\right)^{p}\left(1-\psi_{\rho}\left(\Delta_{i(n, q)}^{n} X\right)\right) \rightarrow\left(\Delta X_{T_{q}}\right)^{p}\left(1-\psi_{\rho}\left(\Delta X_{T_{q}}\right)\right),
$$

pointwise for $p \geq 3$. A straightforward application of the Dubins-Schwarz theorem and Lévy's modulus of continuity leads to

$$
\left(\Delta_{i(n, q)}^{n} X\right)^{2} \mathbb{I}_{\left\{\left|\Delta_{i(n, q)}^{n} X\right|>\alpha \Delta_{n}^{\omega}\right\}}\left(1-\psi_{\rho}\left(\Delta_{i(n, q)}^{n} X\right)\right) \rightarrow\left(\Delta X_{T_{q}}\right)^{2}\left(1-\psi_{\rho}\left(\Delta X_{T_{q}}\right)\right),
$$

for $\alpha>0,0<\omega<1 / 2$ and, hence, we obtain the result.

\section{Tables from the simulation study}


Table 3: Model (D).

\begin{tabular}{rrrrrrrrrrr}
\hline M & R.V.M. & Bias & Std. & 0.100 & 0.050 & R.V.M. & Bias & Std. & 0.100 & 0.050 \\
\hline 78 & RV & -0.31 & 1.19 & 0.150 & 0.100 & TRV & -0.31 & 1.19 & 0.150 & 0.100 \\
& RV2 & -0.31 & 1.19 & 0.150 & 0.100 & TR2V & -0.41 & 1.18 & 0.154 & 0.106 \\
& pRV & -0.28 & 1.07 & 0.121 & 0.078 & R2V & -0.41 & 1.18 & 0.154 & 0.106 \\
& pRV2 & -0.3 & 1.15 & 0.140 & 0.094 & R3V & -0.47 & 1.17 & 0.163 & 0.113 \\
& cRV & -0.31 & 1.19 & 0.150 & 0.100 & R4V & -0.52 & 1.16 & 0.168 & 0.115 \\
\hline 390 & RV & -0.12 & 1.03 & 0.105 & 0.058 & TRV & -0.12 & 1.03 & 0.105 & 0.058 \\
& RV2 & -0.12 & 1.03 & 0.105 & 0.058 & TR2V & -0.17 & 1.03 & 0.111 & 0.064 \\
& pRV & -0.12 & 0.98 & 0.092 & 0.050 & R2V & -0.17 & 1.03 & 0.111 & 0.064 \\
& pRV2 & -0.12 & 1.01 & 0.101 & 0.056 & R3V & -0.2 & 1.03 & 0.113 & 0.067 \\
& cRV & -0.12 & 1.03 & 0.105 & 0.058 & R4V & -0.23 & 1.04 & 0.116 & 0.070 \\
\hline 780 & RV & -0.09 & 1 & 0.102 & 0.050 & TRV & -0.09 & 1 & 0.101 & 0.050 \\
& RV2 & -0.09 & 1 & 0.102 & 0.050 & TR2V & -0.12 & 1 & 0.098 & 0.051 \\
& pRV & -0.09 & 0.98 & 0.091 & 0.044 & R2V & -0.12 & 1 & 0.098 & 0.051 \\
& pRV2 & -0.09 & 0.99 & 0.099 & 0.048 & R3V & -0.14 & 1 & 0.102 & 0.055 \\
& cRV & -0.09 & 1 & 0.102 & 0.050 & R4V & -0.16 & 1 & 0.104 & 0.056 \\
\hline 4680 & RV & -0.07 & 1.01 & 0.105 & 0.054 & TRV & -0.07 & 1.01 & 0.105 & 0.054 \\
& RV2 & -0.07 & 1.01 & 0.105 & 0.054 & TR2V & -0.09 & 1.01 & 0.104 & 0.053 \\
& pRV & -0.07 & 0.99 & 0.101 & 0.054 & R2V & -0.09 & 1.01 & 0.104 & 0.053 \\
& pRV2 & -0.07 & 1 & 0.103 & 0.054 & R3V & -0.09 & 1.01 & 0.105 & 0.054 \\
& cRV & -0.07 & 1.01 & 0.105 & 0.054 & R4V & -0.09 & 1.01 & 0.106 & 0.057 \\
\hline
\end{tabular}


Table 4: Model (JD) with one jump a day, where the percentage of the jump part of the quadratic variation of the total quadratic variation is $15 \%$.

\begin{tabular}{rrrrrrrrrrr}
\hline M & R.V.M. & Bias & Std. & 0.100 & 0.050 & R.V.M. & Bias & Std. & 0.100 & 0.050 \\
\hline 78 & RV & -0.29 & 1.21 & 0.1492 & 0.0976 & TRV & 0.2 & 1.23 & 0.1752 & 0.1094 \\
& RV2 & -0.29 & 1.21 & 0.1502 & 0.0988 & TR2V & -0.17 & 1.12 & 0.1218 & 0.0726 \\
& pRV & -0.25 & 1.1 & 0.1228 & 0.0762 & R2V & -0.03 & 1.14 & 0.126 & 0.0726 \\
& pRV2 & -0.28 & 1.19 & 0.148 & 0.0948 & R3V & -0.19 & 1.11 & 0.1204 & 0.0738 \\
& cRV & -0.29 & 1.26 & 0.1686 & 0.1096 & R4V & -0.27 & 1.1 & 0.1244 & 0.078 \\
\hline 390 & RV & -0.11 & 1.02 & 0.1056 & 0.0532 & TRV & 0.05 & 1.02 & 0.1086 & 0.0578 \\
& RV2 & -0.11 & 1.02 & 0.1068 & 0.0544 & TR2V & -0.1 & 0.98 & 0.0978 & 0.053 \\
& pRV & -0.11 & 1.03 & 0.109 & 0.0556 & R2V & 0.24 & 1.04 & 0.1226 & 0.0678 \\
& pRV2 & -0.11 & 1.06 & 0.1188 & 0.0596 & R3V & 0.04 & 0.99 & 0.0976 & 0.0496 \\
& cRV & -0.11 & 1.13 & 0.1392 & 0.0788 & R4V & -0.03 & 0.98 & 0.0936 & 0.0494 \\
\hline 780 & RV & -0.07 & 1 & 0.095 & 0.0466 & TRV & 0.01 & 0.98 & 0.0972 & 0.0504 \\
& RV2 & -0.07 & 1.01 & 0.096 & 0.0484 & TR2V & -0.07 & 0.97 & 0.088 & 0.0464 \\
& pRV & -0.08 & 1.03 & 0.1046 & 0.0544 & R2V & 0.32 & 1.04 & 0.1338 & 0.0718 \\
& pRV2 & -0.08 & 1.04 & 0.11 & 0.0576 & R3V & 0.1 & 0.97 & 0.0964 & 0.0492 \\
& cRV & -0.08 & 1.12 & 0.1372 & 0.0772 & R4V & 0.03 & 0.97 & 0.0906 & 0.0478 \\
\hline 4680 & RV & -0.04 & 0.98 & 0.0962 & 0.0492 & TRV & -0.04 & 0.98 & 0.0916 & 0.0526 \\
& RV2 & -0.04 & 0.98 & 0.0966 & 0.0498 & TR2V & -0.07 & 0.98 & 0.0922 & 0.047 \\
& pRV & -0.04 & 1.01 & 0.1026 & 0.0544 & R2V & 0.38 & 1.08 & 0.1506 & 0.0896 \\
& pRV2 & -0.04 & 1.01 & 0.1062 & 0.0558 & R3V & 0.11 & 1 & 0.1002 & 0.0494 \\
& cRV & -0.04 & 1.11 & 0.1334 & 0.0784 & R4V & 0.04 & 0.99 & 0.0988 & 0.0474 \\
\hline
\end{tabular}


Table 5: Model (JD) with five jumps a day, where the percentage of the jump part of the quadratic variation of the total quadratic variation is $15 \%$.

\begin{tabular}{rrrrrrrrrrr}
\hline M & R.V.M. & Bias & Std. & 0.100 & 0.050 & R.V.M. & Bias & Std. & 0.100 & 0.050 \\
\hline 78 & RV & -0.3 & 1.19 & 0.1492 & 0.0958 & TRV & 0.68 & 1.18 & 0.2328 & 0.1436 \\
& RV2 & -0.29 & 1.19 & 0.1496 & 0.096 & TR2V & 0.2 & 1.06 & 0.1066 & 0.0498 \\
& pRV & -0.26 & 1.05 & 0.111 & 0.0682 & R2V & 0.22 & 1.06 & 0.1092 & 0.0506 \\
& pRV2 & -0.28 & 1.15 & 0.1388 & 0.0868 & R3V & 0.05 & 1.05 & 0.0912 & 0.0488 \\
& cRV & -0.29 & 1.2 & 0.1538 & 0.0988 & R4V & -0.03 & 1.04 & 0.0898 & 0.0522 \\
\hline 390 & RV & -0.11 & 1.04 & 0.112 & 0.0636 & TRV & 0.96 & 1.12 & 0.2852 & 0.1924 \\
& RV2 & -0.11 & 1.05 & 0.113 & 0.0648 & TR2V & 0.33 & 0.98 & 0.12 & 0.0592 \\
& pRV & -0.1 & 1.02 & 0.1082 & 0.058 & R2V & 0.64 & 1 & 0.183 & 0.0968 \\
& pRV2 & -0.11 & 1.06 & 0.1208 & 0.0664 & R3V & 0.38 & 0.97 & 0.1146 & 0.0566 \\
& cRV & -0.11 & 1.11 & 0.1344 & 0.0772 & R4V & 0.27 & 0.96 & 0.0982 & 0.0498 \\
\hline 780 & RV & -0.07 & 0.98 & 0.0906 & 0.0488 & TRV & 0.71 & 1.05 & 0.21 & 0.1266 \\
& RV2 & -0.07 & 0.99 & 0.0914 & 0.0492 & TR2V & 0.21 & 0.94 & 0.09 & 0.043 \\
& pRV & -0.06 & 1.01 & 0.1002 & 0.0534 & R2V & 0.75 & 0.99 & 0.203 & 0.1154 \\
& pRV2 & -0.06 & 1.03 & 0.1064 & 0.0584 & R3V & 0.43 & 0.94 & 0.1174 & 0.0544 \\
& cRV & -0.07 & 1.08 & 0.1226 & 0.0692 & R4V & 0.31 & 0.94 & 0.099 & 0.045 \\
\hline 4680 & RV & -0.03 & 0.96 & 0.0898 & 0.0412 & TRV & 0.15 & 0.97 & 0.093 & 0.0458 \\
& RV2 & -0.03 & 0.96 & 0.0898 & 0.0412 & TR2V & -0.01 & 0.95 & 0.0854 & 0.0426 \\
& pRV & -0.03 & 1.01 & 0.1068 & 0.055 & R2V & 0.92 & 1.05 & 0.2558 & 0.1648 \\
& pRV2 & -0.03 & 1.01 & 0.1064 & 0.053 & R3V & 0.44 & 0.97 & 0.1198 & 0.0658 \\
& cRV & -0.03 & 1.09 & 0.133 & 0.0742 & R4V & 0.3 & 0.95 & 0.1008 & 0.0524 \\
\hline
\end{tabular}


Table 6: Model (JD) with ten jumps a day, where the percentage of the jump part of the quadratic variation of the total quadratic variation is $15 \%$.

\begin{tabular}{rrrrrrrrrrr}
\hline M & R.V.M. & Bias & Std. & 0.100 & 0.050 & R.V.M. & Bias & Std. & 0.100 & 0.050 \\
\hline 78 & RV & -0.25 & 1.17 & 0.1426 & 0.0884 & TRV & 0.77 & 1.1 & 0.2272 & 0.1372 \\
& RV2 & -0.25 & 1.17 & 0.1432 & 0.0886 & TR2V & 0.34 & 1.02 & 0.1094 & 0.049 \\
& pRV & -0.22 & 1.04 & 0.1048 & 0.0636 & R2V & 0.35 & 1.02 & 0.1108 & 0.05 \\
& pRV2 & -0.24 & 1.13 & 0.1308 & 0.0808 & R3V & 0.18 & 1.01 & 0.09 & 0.0442 \\
& cRV & -0.24 & 1.17 & 0.1438 & 0.0892 & R4V & 0.09 & 1 & 0.087 & 0.0444 \\
\hline \multirow{2}{*}{390} & RV & -0.14 & 1.05 & 0.1192 & 0.0658 & TRV & 1.56 & 1.16 & 0.4688 & 0.3574 \\
& RV2 & -0.14 & 1.06 & 0.119 & 0.0664 & TR2V & 0.68 & 0.97 & 0.176 & 0.0892 \\
& pRV & -0.13 & 0.99 & 0.101 & 0.0532 & R2V & 0.83 & 0.97 & 0.2244 & 0.1256 \\
& pRV2 & -0.14 & 1.05 & 0.1206 & 0.0644 & R3V & 0.56 & 0.95 & 0.1456 & 0.071 \\
& cRV & -0.14 & 1.08 & 0.1308 & 0.0724 & R4V & 0.45 & 0.95 & 0.1212 & 0.0572 \\
\hline 780 & RV & -0.1 & 1.02 & 0.1088 & 0.061 & TRV & 1.5 & 1.16 & 0.455 & 0.3488 \\
& RV2 & -0.1 & 1.03 & 0.1108 & 0.0624 & TR2V & 0.58 & 0.96 & 0.152 & 0.0814 \\
& pRV & -0.09 & 1.02 & 0.1066 & 0.0564 & R2V & 1 & 0.98 & 0.2788 & 0.167 \\
& pRV2 & -0.09 & 1.05 & 0.1176 & 0.0654 & R3V & 0.65 & 0.95 & 0.157 & 0.0834 \\
& cRV & -0.1 & 1.09 & 0.1322 & 0.077 & R4V & 0.51 & 0.94 & 0.1226 & 0.066 \\
\hline 4680 & RV & -0.02 & 1 & 0.1026 & 0.0464 & TRV & 0.54 & 1.03 & 0.1672 & 0.0984 \\
& RV2 & -0.02 & 1 & 0.1026 & 0.0456 & TR2V & 0.13 & 0.97 & 0.0952 & 0.0464 \\
& pRV & -0.02 & 1.06 & 0.125 & 0.0634 & R2V & 1.3 & 1.04 & 0.382 & 0.2714 \\
& pRV2 & -0.02 & 1.06 & 0.1232 & 0.0626 & R3V & 0.71 & 0.97 & 0.1784 & 0.1028 \\
& cRV & -0.02 & 1.12 & 0.1434 & 0.0828 & R4V & 0.52 & 0.96 & 0.1342 & 0.0724 \\
\hline
\end{tabular}


Table 7: Model (IAP) with $\alpha=0.1$, where the percentage of the jump part of the quadratic variation of the total quadratic variation is $15 \%$.

\begin{tabular}{rrrrrrrrrrr}
\hline M & R.V.M. & Bias & Std. & 0.100 & 0.050 & R.V.M. & Bias & Std. & 0.100 & 0.050 \\
\hline 78 & RV & -0.3 & 1.19 & 0.1488 & 0.1004 & TRV & 0.41 & 1.22 & 0.198 & 0.1256 \\
& RV2 & -0.3 & 1.19 & 0.1492 & 0.1012 & TR2V & 0.03 & 1.11 & 0.1182 & 0.0702 \\
& pRV & -0.26 & 1.07 & 0.1172 & 0.0732 & R2V & 0.11 & 1.14 & 0.1326 & 0.0776 \\
& pRV2 & -0.28 & 1.15 & 0.1436 & 0.0918 & R3V & -0.04 & 1.1 & 0.1158 & 0.07 \\
& cRV & -0.29 & 1.22 & 0.1588 & 0.1068 & R4V & -0.13 & 1.09 & 0.119 & 0.0722 \\
\hline 390 & RV & -0.13 & 1.09 & 0.1094 & 0.0616 & TRV & 0.6 & 1.11 & 0.198 & 0.1178 \\
& RV2 & -0.13 & 1.1 & 0.111 & 0.0634 & TR2V & 0.18 & 1 & 0.1072 & 0.0536 \\
& pRV & -0.12 & 1.08 & 0.1052 & 0.0636 & R2V & 0.47 & 1.07 & 0.1684 & 0.0954 \\
& pRV2 & -0.13 & 1.12 & 0.1158 & 0.069 & R3V & 0.25 & 1 & 0.1134 & 0.0536 \\
& cRV & -0.13 & 1.18 & 0.135 & 0.0848 & R4V & 0.15 & 0.99 & 0.102 & 0.046 \\
\hline 780 & RV & -0.1 & 1.12 & 0.1014 & 0.0518 & TRV & 0.56 & 1.07 & 0.1736 & 0.1026 \\
& RV2 & -0.1 & 1.12 & 0.1024 & 0.052 & TR2V & 0.18 & 0.97 & 0.1008 & 0.0486 \\
& pRV & -0.1 & 1.13 & 0.1054 & 0.0568 & R2V & 0.59 & 1.08 & 0.189 & 0.1134 \\
& pRV2 & -0.1 & 1.16 & 0.1146 & 0.0608 & R3V & 0.32 & 0.99 & 0.118 & 0.0546 \\
& cRV & -0.11 & 1.21 & 0.1318 & 0.0758 & R4V & 0.22 & 0.97 & 0.0982 & 0.0472 \\
\hline 4680 & RV & -0.06 & 1.08 & 0.09 & 0.044 & TRV & 0.38 & 1 & 0.127 & 0.0646 \\
& RV2 & -0.06 & 1.09 & 0.0898 & 0.044 & TR2V & 0.1 & 0.96 & 0.084 & 0.0432 \\
& pRV & -0.06 & 1.15 & 0.101 & 0.051 & R2V & 0.79 & 1.12 & 0.2324 & 0.1522 \\
& pRV2 & -0.06 & 1.15 & 0.101 & 0.0518 & R3V & 0.39 & 0.98 & 0.1256 & 0.0616 \\
& cRV & -0.06 & 1.24 & 0.1302 & 0.073 & R4V & 0.28 & 0.97 & 0.1026 & 0.0474 \\
\hline
\end{tabular}


Table 8: Model (IAP) with $\alpha=0.5$, where the percentage of the jump part of the quadratic variation of the total quadratic variation is $15 \%$.

\begin{tabular}{rrrrrrrrrrr}
\hline M & R.V.M. & Bias & Std. & 0.100 & 0.050 & R.V.M. & Bias & Std. & 0.100 & 0.050 \\
\hline 78 & RV & -0.27 & 1.17 & 0.1476 & 0.0916 & TRV & 0.64 & 1.14 & 0.2096 & 0.122 \\
& RV2 & -0.27 & 1.17 & 0.1476 & 0.092 & TR2V & 0.26 & 1.03 & 0.111 & 0.0518 \\
& pRV & -0.25 & 1.04 & 0.1092 & 0.0644 & R2V & 0.27 & 1.04 & 0.1154 & 0.054 \\
& pRV2 & -0.26 & 1.13 & 0.136 & 0.0818 & R3V & 0.12 & 1.02 & 0.0996 & 0.0516 \\
& cRV & -0.27 & 1.18 & 0.1492 & 0.093 & R4V & 0.03 & 1.01 & 0.0968 & 0.0522 \\
\hline \multirow{2}{*}{390} & RV & -0.13 & 1.06 & 0.1186 & 0.0658 & TRV & 1.35 & 1.13 & 0.4028 & 0.2964 \\
& RV2 & -0.13 & 1.06 & 0.1192 & 0.0666 & TR2V & 0.71 & 0.97 & 0.1862 & 0.0994 \\
& pRV & -0.12 & 1.02 & 0.1062 & 0.0576 & R2V & 0.87 & 1 & 0.2338 & 0.141 \\
& pRV2 & -0.13 & 1.06 & 0.1192 & 0.0664 & R3V & 0.63 & 0.97 & 0.1584 & 0.0802 \\
& cRV & -0.13 & 1.1 & 0.1292 & 0.075 & R4V & 0.53 & 0.96 & 0.1346 & 0.0664 \\
\hline 780 & RV & -0.11 & 1.02 & 0.1064 & 0.0602 & TRV & 1.56 & 1.14 & 0.473 & 0.3588 \\
& RV2 & -0.11 & 1.03 & 0.1076 & 0.0612 & TR2V & 0.8 & 0.97 & 0.2006 & 0.1134 \\
& pRV & -0.1 & 1.01 & 0.1038 & 0.0564 & R2V & 1.09 & 1.02 & 0.3102 & 0.2046 \\
& pRV2 & -0.11 & 1.04 & 0.1128 & 0.066 & R3V & 0.78 & 0.97 & 0.2052 & 0.1114 \\
& cRV & -0.11 & 1.08 & 0.1218 & 0.0748 & R4V & 0.65 & 0.96 & 0.1612 & 0.0834 \\
\hline 4680 & RV & -0.03 & 0.98 & 0.1004 & 0.0474 & TRV & 1.79 & 1.11 & 0.5416 & 0.4264 \\
& RV2 & -0.03 & 0.98 & 0.101 & 0.0474 & TR2V & 0.86 & 0.95 & 0.2096 & 0.1276 \\
& pRV & -0.03 & 1.01 & 0.1134 & 0.0568 & R2V & 1.71 & 1.06 & 0.5258 & 0.407 \\
& pRV2 & -0.03 & 1.02 & 0.117 & 0.0592 & R3V & 1.13 & 0.96 & 0.3064 & 0.2018 \\
& cRV & -0.03 & 1.08 & 0.1326 & 0.0734 & R4V & 0.93 & 0.94 & 0.2348 & 0.1396 \\
\hline
\end{tabular}


Table 9: Model (IAP) with $\alpha=0.9$, where the percentage of the jump part of the quadratic variation of the total quadratic variation is $15 \%$.

\begin{tabular}{rrrrrrrrrrr}
\hline M & R.V.M. & Bias & Std. & 0.100 & 0.050 & R.V.M. & Bias & Std. & 0.100 & 0.050 \\
\hline 78 & RV & -0.29 & 1.15 & 0.1384 & 0.09 & TRV & 0.68 & 1.01 & 0.1822 & 0.1006 \\
& RV2 & -0.29 & 1.15 & 0.1384 & 0.09 & TR2V & 0.42 & 0.99 & 0.1214 & 0.052 \\
& pRV & -0.27 & 1.04 & 0.11 & 0.0652 & R2V & 0.42 & 0.99 & 0.1216 & 0.0522 \\
& pRV2 & -0.28 & 1.11 & 0.129 & 0.0814 & R3V & 0.29 & 0.99 & 0.1012 & 0.047 \\
& cRV & -0.29 & 1.15 & 0.1386 & 0.09 & R4V & 0.21 & 0.99 & 0.097 & 0.0482 \\
\hline \multirow{2}{*}{390} & RV & -0.14 & 1.04 & 0.117 & 0.061 & TRV & 1.86 & 1.01 & 0.5914 & 0.4686 \\
& RV2 & -0.14 & 1.04 & 0.1172 & 0.061 & TR2V & 1.37 & 0.92 & 0.408 & 0.2762 \\
& pRV & -0.13 & 0.99 & 0.099 & 0.05 & R2V & 1.38 & 0.92 & 0.413 & 0.2792 \\
& pRV2 & -0.13 & 1.02 & 0.1108 & 0.057 & R3V & 1.18 & 0.92 & 0.3302 & 0.206 \\
& cRV & -0.14 & 1.04 & 0.1184 & 0.0616 & R4V & 1.07 & 0.93 & 0.2874 & 0.1704 \\
\hline 780 & RV & -0.09 & 1.02 & 0.1072 & 0.0562 & TRV & 2.66 & 1.1 & 0.8218 & 0.7366 \\
& RV2 & -0.09 & 1.02 & 0.1076 & 0.0562 & TR2V & 1.89 & 0.96 & 0.6158 & 0.4866 \\
& pRV & -0.09 & 0.98 & 0.0928 & 0.0476 & R2V & 1.92 & 0.96 & 0.6308 & 0.5006 \\
& pRV2 & -0.09 & 1.01 & 0.1024 & 0.0542 & R3V & 1.65 & 0.94 & 0.5164 & 0.3788 \\
& cRV & -0.09 & 1.03 & 0.109 & 0.0568 & R4V & 1.5 & 0.93 & 0.454 & 0.3178 \\
\hline 4680 & RV & -0.03 & 1.01 & 0.104 & 0.0522 & TRV & 5.41 & 1.52 & 0.994 & 0.9902 \\
& RV2 & -0.03 & 1.01 & 0.1042 & 0.053 & TR2V & 3.33 & 1.07 & 0.9354 & 0.8956 \\
& pRV & -0.03 & 1 & 0.0994 & 0.05 & R2V & 3.73 & 1.1 & 0.9638 & 0.9418 \\
& pRV2 & -0.03 & 1.03 & 0.1082 & 0.054 & R3V & 3.02 & 1.02 & 0.9088 & 0.85 \\
& cRV & -0.03 & 1.05 & 0.1148 & 0.0596 & R4V & 2.71 & 1 & 0.8556 & 0.7806 \\
\hline
\end{tabular}


Table 10: Model (IFAJ) with five jumps a day.

\begin{tabular}{rrrrrrrrrrr}
\hline M & R.V.M. & Bias & Std. & 0.100 & 0.050 & R.V.M. & Bias & Std. & 0.100 & 0.050 \\
\hline 78 & RV & -0.3 & 1.17 & 0.1442 & 0.0956 & TRV & 0.46 & 1.15 & 0.1798 & 0.1078 \\
& RV2 & -0.3 & 1.17 & 0.1456 & 0.096 & TR2V & 0.06 & 1.06 & 0.098 & 0.058 \\
& pRV & -0.26 & 1.06 & 0.1126 & 0.0712 & R2V & 0.16 & 1.08 & 0.1088 & 0.0616 \\
& pRV2 & -0.29 & 1.14 & 0.1362 & 0.0898 & R3V & 0 & 1.05 & 0.0994 & 0.055 \\
& cRV & -0.29 & 1.21 & 0.1534 & 0.103 & R4V & -0.08 & 1.04 & 0.1048 & 0.057 \\
\hline 390 & RV & -0.12 & 1.03 & 0.1166 & 0.0572 & TRV & 0.49 & 1.04 & 0.1608 & 0.0908 \\
& RV2 & -0.12 & 1.03 & 0.1178 & 0.0596 & TR2V & 0.11 & 0.98 & 0.0966 & 0.0522 \\
& pRV & -0.11 & 1.03 & 0.1178 & 0.0602 & R2V & 0.53 & 1.04 & 0.1634 & 0.0944 \\
& pRV2 & -0.12 & 1.06 & 0.1264 & 0.0682 & R3V & 0.27 & 0.99 & 0.1126 & 0.053 \\
& cRV & -0.12 & 1.12 & 0.1438 & 0.0818 & R4V & 0.17 & 0.98 & 0.1002 & 0.0472 \\
\hline 780 & RV & -0.08 & 1.02 & 0.1024 & 0.0506 & TRV & 0.36 & 1.01 & 0.1264 & 0.068 \\
& RV2 & -0.08 & 1.02 & 0.1038 & 0.0506 & TR2V & 0.09 & 0.97 & 0.0912 & 0.0446 \\
& pRV & -0.08 & 1.06 & 0.1166 & 0.0596 & R2V & 0.67 & 1.05 & 0.201 & 0.1144 \\
& pRV2 & -0.09 & 1.07 & 0.1196 & 0.0628 & R3V & 0.37 & 0.99 & 0.1208 & 0.0608 \\
& cRV & -0.09 & 1.13 & 0.138 & 0.0794 & R4V & 0.27 & 0.99 & 0.1084 & 0.0502 \\
\hline 4680 & RV & -0.03 & 1.12 & 0.0996 & 0.047 & TRV & 0.11 & 0.99 & 0.1 & 0.05 \\
& RV2 & -0.03 & 1.12 & 0.1002 & 0.0468 & TR2V & -0.01 & 0.97 & 0.0924 & 0.0468 \\
& pRV & -0.03 & 1.19 & 0.1178 & 0.0592 & R2V & 0.83 & 1.09 & 0.2438 & 0.159 \\
& pRV2 & -0.03 & 1.19 & 0.1178 & 0.059 & R3V & 0.39 & 1 & 0.124 & 0.0692 \\
& cRV & -0.03 & 1.28 & 0.1442 & 0.0822 & R4V & 0.27 & 0.98 & 0.1102 & 0.0536 \\
\hline
\end{tabular}


Table 11: Model (IFAJ) with ten jumps a day.

\begin{tabular}{rrrrrrrrrrr}
\hline M & R.V.M. & Bias & Std. & 0.100 & 0.050 & R.V.M. & Bias & Std. & 0.100 & 0.050 \\
\hline 78 & RV & -0.31 & 1.16 & 0.1444 & 0.0972 & TRV & 0.26 & 1.12 & 0.144 & 0.0838 \\
& RV2 & -0.31 & 1.16 & 0.1446 & 0.0974 & TR2V & 0 & 1.09 & 0.1126 & 0.0636 \\
& pRV & -0.28 & 1.04 & 0.115 & 0.074 & R2V & 0.01 & 1.1 & 0.1136 & 0.0642 \\
& pRV2 & -0.3 & 1.12 & 0.1358 & 0.09 & R3V & -0.09 & 1.09 & 0.1084 & 0.0648 \\
& cRV & -0.31 & 1.17 & 0.1458 & 0.098 & R4V & -0.16 & 1.08 & 0.1146 & 0.0684 \\
\hline 390 & RV & -0.16 & 1.04 & 0.1144 & 0.0586 & TRV & 0.66 & 1.05 & 0.2 & 0.1152 \\
& RV2 & -0.16 & 1.04 & 0.1152 & 0.0592 & TR2V & 0.27 & 0.98 & 0.1092 & 0.0522 \\
& pRV & -0.15 & 1 & 0.1026 & 0.0534 & R2V & 0.41 & 1 & 0.1332 & 0.0666 \\
& pRV2 & -0.16 & 1.04 & 0.1146 & 0.0598 & R3V & 0.25 & 0.98 & 0.1038 & 0.0472 \\
& cRV & -0.16 & 1.06 & 0.1238 & 0.0654 & R4V & 0.17 & 0.98 & 0.095 & 0.0446 \\
\hline 780 & RV & -0.11 & 1 & 0.1044 & 0.0574 & TRV & 0.69 & 1.02 & 0.1918 & 0.1106 \\
& RV2 & -0.11 & 1.01 & 0.1052 & 0.0582 & TR2V & 0.26 & 0.96 & 0.099 & 0.0472 \\
& pRV & -0.11 & 1 & 0.1042 & 0.0532 & R2V & 0.53 & 0.98 & 0.1442 & 0.0732 \\
& pRV2 & -0.11 & 1.02 & 0.1112 & 0.0604 & R3V & 0.33 & 0.97 & 0.105 & 0.0522 \\
& cRV & -0.11 & 1.05 & 0.1178 & 0.0676 & R4V & 0.24 & 0.97 & 0.1012 & 0.0478 \\
\hline 4680 & RV & -0.04 & 1 & 0.0984 & 0.0514 & TRV & 0.39 & 1 & 0.1296 & 0.0756 \\
& RV2 & -0.04 & 1 & 0.0986 & 0.0512 & TR2V & 0.1 & 0.97 & 0.0918 & 0.0464 \\
& pRV & -0.04 & 1.03 & 0.1108 & 0.0582 & R2V & 0.78 & 1.01 & 0.214 & 0.1316 \\
& pRV2 & -0.04 & 1.04 & 0.1118 & 0.06 & R3V & 0.44 & 0.96 & 0.1242 & 0.0622 \\
& cRV & -0.05 & 1.07 & 0.122 & 0.0684 & R4V & 0.32 & 0.96 & 0.1026 & 0.0566 \\
\hline
\end{tabular}


Table 12: Model (CFAJ) with five jumps a day.

\begin{tabular}{rrrrrrrrrrr}
\hline M & R.V.M. & Bias & Std. & 0.100 & 0.050 & R.V.M. & Bias & Std. & 0.100 & 0.050 \\
\hline 78 & RV & -0.31 & 1.2 & 0.1468 & 0.098 & TRV & 0.38 & 1.11 & 0.1578 & 0.0866 \\
& RV2 & -0.31 & 1.21 & 0.1474 & 0.098 & TR2V & 0.06 & 1.06 & 0.0954 & 0.0524 \\
& pRV & -0.26 & 1.08 & 0.1172 & 0.0746 & R2V & 0.17 & 1.08 & 0.1072 & 0.0546 \\
& pRV2 & -0.29 & 1.17 & 0.1412 & 0.0908 & R3V & 0.01 & 1.06 & 0.092 & 0.055 \\
& cRV & -0.3 & 1.24 & 0.159 & 0.1058 & R4V & -0.07 & 1.05 & 0.0938 & 0.0576 \\
\hline 390 & RV & -0.14 & 1.03 & 0.1082 & 0.058 & TRV & 0.45 & 1.02 & 0.1446 & 0.084 \\
& RV2 & -0.14 & 1.04 & 0.11 & 0.0604 & TR2V & 0.12 & 0.97 & 0.0924 & 0.0482 \\
& pRV & -0.13 & 1.05 & 0.1164 & 0.0606 & R2V & 0.57 & 1.03 & 0.1642 & 0.093 \\
& pRV2 & -0.13 & 1.07 & 0.126 & 0.066 & R3V & 0.32 & 0.98 & 0.1126 & 0.0532 \\
& cRV & -0.13 & 1.13 & 0.141 & 0.081 & R4V & 0.22 & 0.97 & 0.0984 & 0.0498 \\
\hline 780 & RV & -0.09 & 0.99 & 0.096 & 0.052 & TRV & 0.36 & 0.99 & 0.1186 & 0.0646 \\
& RV2 & -0.09 & 0.99 & 0.0962 & 0.0522 & TR2V & 0.08 & 0.95 & 0.086 & 0.0416 \\
& pRV & -0.09 & 1.03 & 0.1096 & 0.063 & R2V & 0.68 & 1.01 & 0.191 & 0.1056 \\
& pRV2 & -0.1 & 1.04 & 0.1138 & 0.0654 & R3V & 0.39 & 0.95 & 0.1118 & 0.0478 \\
& cRV & -0.1 & 1.11 & 0.1344 & 0.0816 & R4V & 0.28 & 0.94 & 0.0976 & 0.0422 \\
\hline 4680 & RV & -0.05 & 0.96 & 0.0846 & 0.0418 & TRV & 0.09 & 0.96 & 0.088 & 0.0428 \\
& RV2 & -0.05 & 0.96 & 0.0852 & 0.0418 & TR2V & -0.03 & 0.95 & 0.0866 & 0.0428 \\
& pRV & -0.05 & 1.02 & 0.1062 & 0.0546 & R2V & 0.85 & 1.05 & 0.2396 & 0.1516 \\
& pRV2 & -0.05 & 1.02 & 0.1048 & 0.0536 & R3V & 0.41 & 0.97 & 0.1198 & 0.0578 \\
& cRV & -0.05 & 1.11 & 0.1344 & 0.074 & R4V & 0.28 & 0.96 & 0.0972 & 0.0504 \\
\hline
\end{tabular}


Table 13: Model (CFAJ) with ten jumps a day.

\begin{tabular}{rrrrrrrrrrr}
\hline M & R.V.M. & Bias & Std. & 0.100 & 0.050 & R.V.M. & Bias & Std. & 0.100 & 0.050 \\
\hline 78 & RV & -0.27 & 1.15 & 0.1428 & 0.088 & TRV & 0.26 & 1.08 & 0.1298 & 0.0692 \\
& RV2 & -0.27 & 1.16 & 0.143 & 0.0882 & TR2V & 0.02 & 1.06 & 0.1014 & 0.0558 \\
& pRV & -0.24 & 1.04 & 0.1114 & 0.0694 & R2V & 0.04 & 1.06 & 0.1016 & 0.055 \\
& pRV2 & -0.26 & 1.12 & 0.1328 & 0.0814 & R3V & -0.06 & 1.05 & 0.1028 & 0.0578 \\
& cRV & -0.26 & 1.16 & 0.1454 & 0.0916 & R4V & -0.13 & 1.05 & 0.1062 & 0.063 \\
\hline 390 & RV & -0.14 & 1.03 & 0.112 & 0.061 & TRV & 0.64 & 1.02 & 0.1788 & 0.0998 \\
& RV2 & -0.14 & 1.03 & 0.1118 & 0.0612 & TR2V & 0.28 & 0.97 & 0.1046 & 0.0492 \\
& pRV & -0.13 & 1 & 0.1012 & 0.0522 & R2V & 0.43 & 0.99 & 0.1324 & 0.0644 \\
& pRV2 & -0.13 & 1.04 & 0.1134 & 0.0606 & R3V & 0.28 & 0.98 & 0.1036 & 0.0514 \\
& cRV & -0.14 & 1.06 & 0.1216 & 0.0672 & R4V & 0.21 & 0.97 & 0.0964 & 0.0488 \\
\hline 780 & RV & -0.09 & 1.01 & 0.103 & 0.056 & TRV & 0.67 & 1.02 & 0.1886 & 0.1098 \\
& RV2 & -0.09 & 1.01 & 0.1038 & 0.0562 & TR2V & 0.28 & 0.96 & 0.1032 & 0.0458 \\
& pRV & -0.09 & 1.01 & 0.1 & 0.053 & R2V & 0.57 & 0.98 & 0.1536 & 0.0782 \\
& pRV2 & -0.09 & 1.03 & 0.1094 & 0.0582 & R3V & 0.37 & 0.96 & 0.1118 & 0.0542 \\
& cRV & -0.09 & 1.06 & 0.1182 & 0.0646 & R4V & 0.29 & 0.96 & 0.104 & 0.0496 \\
\hline 4680 & RV & -0.02 & 1 & 0.101 & 0.0512 & TRV & 0.4 & 1.01 & 0.138 & 0.0738 \\
& RV2 & -0.02 & 1 & 0.1014 & 0.0514 & TR2V & 0.12 & 0.98 & 0.1004 & 0.0492 \\
& pRV & -0.02 & 1.04 & 0.1108 & 0.0586 & R2V & 0.84 & 1.02 & 0.228 & 0.1464 \\
& pRV2 & -0.02 & 1.04 & 0.1128 & 0.0608 & R3V & 0.49 & 0.98 & 0.1366 & 0.075 \\
& cRV & -0.02 & 1.08 & 0.124 & 0.0676 & R4V & 0.36 & 0.97 & 0.1158 & 0.0616 \\
\hline
\end{tabular}


Table 14: Model (BNSIJ) without jumps in the price.

\begin{tabular}{rrrrrrrrrrr}
\hline M & R.V.M. & Bias & Std. & 0.100 & 0.050 & R.V.M. & Bias & Std. & 0.100 & 0.050 \\
\hline \multirow{2}{*}{78} & RV & -0.25 & 1.18 & 0.1424 & 0.0926 & TRV & -0.3 & 1.21 & 0.1508 & 0.1 \\
& RV2 & -0.26 & 1.18 & 0.1426 & 0.0926 & TR2V & -0.4 & 1.21 & 0.1514 & 0.102 \\
& pRV & -0.23 & 1.08 & 0.1178 & 0.0696 & R2V & -0.36 & 1.19 & 0.1444 & 0.0948 \\
& pRV2 & -0.25 & 1.15 & 0.1368 & 0.0852 & R3V & -0.43 & 1.17 & 0.1488 & 0.0968 \\
& cRV & -0.25 & 1.19 & 0.1446 & 0.0936 & R4V & -0.47 & 1.16 & 0.153 & 0.0962 \\
\hline \multirow{2}{*}{390} & RV & -0.11 & 1.03 & 0.11 & 0.0622 & TRV & -0.19 & 1.15 & 0.1256 & 0.0774 \\
& RV2 & -0.11 & 1.03 & 0.1098 & 0.0622 & TR2V & -0.21 & 1.12 & 0.1264 & 0.0746 \\
& pRV & -0.1 & 0.99 & 0.0998 & 0.0542 & R2V & -0.14 & 1.03 & 0.1132 & 0.0622 \\
& pRV2 & -0.11 & 1.02 & 0.1068 & 0.0606 & R3V & -0.17 & 1.03 & 0.114 & 0.062 \\
& cRV & -0.11 & 1.04 & 0.1108 & 0.0632 & R4V & -0.19 & 1.03 & 0.1118 & 0.0632 \\
\hline \multirow{2}{*}{780} & RV & -0.08 & 1.01 & 0.1046 & 0.054 & TRV & -0.19 & 1.24 & 0.1252 & 0.0736 \\
& RV2 & -0.08 & 1 & 0.1046 & 0.0534 & TR2V & -0.2 & 1.19 & 0.123 & 0.0678 \\
& pRV & -0.08 & 0.98 & 0.095 & 0.0476 & R2V & -0.11 & 1.01 & 0.1048 & 0.0506 \\
& pRV2 & -0.08 & 1 & 0.1018 & 0.052 & R3V & -0.13 & 1.01 & 0.1048 & 0.0556 \\
& cRV & -0.08 & 1.01 & 0.1062 & 0.0552 & R4V & -0.15 & 1 & 0.1038 & 0.0534 \\
\hline \multirow{2}{*}{4680} & RV & -0.04 & 1 & 0.0996 & 0.051 & TRV & -0.26 & 1.8 & 0.1262 & 0.0768 \\
& RV2 & -0.04 & 1 & 0.0992 & 0.0508 & TR2V & -0.24 & 1.65 & 0.1222 & 0.0774 \\
& pRV & -0.04 & 0.99 & 0.0982 & 0.049 & R2V & -0.05 & 0.99 & 0.0982 & 0.0526 \\
& pRV2 & -0.04 & 1 & 0.1004 & 0.051 & R3V & -0.05 & 0.99 & 0.1008 & 0.055 \\
& $\mathrm{cRV}$ & -0.04 & 1 & 0.1012 & 0.0516 & R4V & -0.05 & 0.99 & 0.1018 & 0.053 \\
\hline
\end{tabular}


Table 15: Model (BNSIJ) with one jump in the price, where the percentage of the jump part of the $\mathrm{QV}$ is $15 \%$.

\begin{tabular}{rrrrrrrrrrr}
\hline M & R.V.M. & Bias & Std. & 0.100 & 0.050 & R.V.M. & Bias & Std. & 0.100 & 0.050 \\
\hline 78 & RV & -0.26 & 1.2 & 0.144 & 0.094 & TRV & 0.3 & 1.32 & 0.2078 & 0.1382 \\
& RV2 & -0.26 & 1.21 & 0.1452 & 0.0956 & TR2V & -0.14 & 1.12 & 0.1164 & 0.0674 \\
& pRV & -0.23 & 1.1 & 0.1132 & 0.0688 & R2V & 0.03 & 1.12 & 0.1178 & 0.0624 \\
& pRV2 & -0.25 & 1.18 & 0.14 & 0.0892 & R3V & -0.14 & 1.08 & 0.1046 & 0.0606 \\
& cRV & -0.26 & 1.26 & 0.1636 & 0.1094 & R4V & -0.23 & 1.07 & 0.111 & 0.0658 \\
\hline 390 & RV & -0.14 & 1.12 & 0.1048 & 0.058 & TRV & 0.01 & 1.17 & 0.1376 & 0.0826 \\
& RV2 & -0.14 & 1.13 & 0.1074 & 0.059 & TR2V & -0.15 & 1.09 & 0.1142 & 0.063 \\
& pRV & -0.13 & 1.13 & 0.1094 & 0.0576 & R2V & 0.27 & 1.06 & 0.1356 & 0.0716 \\
& pRV2 & -0.14 & 1.16 & 0.1174 & 0.0654 & R3V & 0.05 & 1.01 & 0.098 & 0.0494 \\
& cRV & -0.14 & 1.25 & 0.1472 & 0.0898 & R4V & -0.02 & 1 & 0.0988 & 0.0492 \\
\hline 780 & RV & -0.09 & 1.05 & 0.1068 & 0.0588 & TRV & -0.06 & 1.23 & 0.1318 & 0.0744 \\
& RV2 & -0.09 & 1.06 & 0.1072 & 0.0602 & TR2V & -0.16 & 1.14 & 0.1104 & 0.0646 \\
& pRV & -0.09 & 1.09 & 0.1186 & 0.0656 & R2V & 0.35 & 1.06 & 0.1412 & 0.0816 \\
& pRV2 & -0.09 & 1.1 & 0.1234 & 0.0714 & R3V & 0.12 & 0.99 & 0.102 & 0.0474 \\
& cRV & -0.1 & 1.2 & 0.151 & 0.0946 & R4V & 0.04 & 0.98 & 0.0932 & 0.0452 \\
\hline 4680 & RV & -0.04 & 1.01 & 0.0982 & 0.0502 & TRV & -0.24 & 1.75 & 0.122 & 0.073 \\
& RV2 & -0.04 & 1.01 & 0.0992 & 0.0506 & TR2V & -0.25 & 1.61 & 0.118 & 0.0718 \\
& pRV & -0.04 & 1.04 & 0.1114 & 0.058 & R2V & 0.42 & 1.09 & 0.1588 & 0.0948 \\
& pRV2 & -0.04 & 1.05 & 0.112 & 0.058 & R3V & 0.12 & 1 & 0.1008 & 0.0504 \\
& $\mathrm{cRV}$ & -0.04 & 1.17 & 0.1534 & 0.0888 & R4V & 0.04 & 0.99 & 0.0968 & 0.0464 \\
\hline
\end{tabular}


Table 16: Model (BNSIJ) with five jumps in the price, where the percentage of the jump part of the QV is $15 \%$.

\begin{tabular}{rrrrrrrrrrr}
\hline M & R.V.M. & Bias & Std. & 0.100 & 0.050 & R.V.M. & Bias & Std. & 0.100 & 0.050 \\
\hline 78 & RV & -0.28 & 1.21 & 0.1616 & 0.1062 & TRV & 0.87 & 1.34 & 0.3092 & 0.2188 \\
& RV2 & -0.28 & 1.21 & 0.1612 & 0.1066 & TR2V & 0.27 & 1.12 & 0.1352 & 0.0708 \\
& pRV & -0.24 & 1.05 & 0.1162 & 0.0674 & R2V & 0.32 & 1.08 & 0.1304 & 0.065 \\
& pRV2 & -0.26 & 1.16 & 0.1468 & 0.0944 & R3V & 0.13 & 1.05 & 0.102 & 0.05 \\
& cRV & -0.28 & 1.23 & 0.1658 & 0.1096 & R4V & 0.03 & 1.04 & 0.101 & 0.0512 \\
\hline 390 & RV & -0.13 & 1.05 & 0.1168 & 0.0648 & TRV & 1.14 & 1.41 & 0.3838 & 0.2908 \\
& RV2 & -0.13 & 1.06 & 0.1186 & 0.0664 & TR2V & 0.32 & 1.11 & 0.1358 & 0.0754 \\
& pRV & -0.11 & 1.02 & 0.1066 & 0.0578 & R2V & 0.72 & 1.02 & 0.2058 & 0.1162 \\
& pRV2 & -0.12 & 1.07 & 0.125 & 0.068 & R3V & 0.43 & 0.97 & 0.121 & 0.053 \\
& cRV & -0.13 & 1.13 & 0.1466 & 0.0876 & R4V & 0.31 & 0.96 & 0.0958 & 0.0468 \\
\hline 780 & RV & -0.09 & 1 & 0.0986 & 0.0488 & TRV & 0.8 & 1.4 & 0.2886 & 0.1984 \\
& RV2 & -0.09 & 1.01 & 0.0998 & 0.0496 & TR2V & 0.17 & 1.18 & 0.126 & 0.0678 \\
& pRV & -0.08 & 1.03 & 0.104 & 0.053 & R2V & 0.85 & 1.04 & 0.243 & 0.1446 \\
& pRV2 & -0.09 & 1.05 & 0.1142 & 0.0586 & R3V & 0.48 & 0.98 & 0.137 & 0.0674 \\
& cRV & -0.09 & 1.13 & 0.139 & 0.078 & R4V & 0.35 & 0.97 & 0.1086 & 0.0544 \\
\hline 4680 & RV & -0.03 & 0.98 & 0.0902 & 0.0436 & TRV & 0.01 & 1.84 & 0.1316 & 0.0786 \\
& RV2 & -0.03 & 0.98 & 0.09 & 0.0432 & TR2V & -0.17 & 1.65 & 0.1086 & 0.0672 \\
& pRV & -0.03 & 1.04 & 0.1146 & 0.0578 & R2V & 1.05 & 1.07 & 0.2974 & 0.2 \\
& pRV2 & -0.03 & 1.04 & 0.1106 & 0.0556 & R3V & 0.5 & 0.97 & 0.1352 & 0.0724 \\
& cRV & -0.03 & 1.16 & 0.1512 & 0.0848 & R4V & 0.34 & 0.95 & 0.1072 & 0.0546 \\
\hline
\end{tabular}


Table 17: Model (BNSIJ) with ten jumps in the price, where the percentage of the jump part of the QV is $15 \%$.

\begin{tabular}{rrrrrrrrrrr}
\hline M & R.V.M. & Bias & Std. & 0.100 & 0.050 & R.V.M. & Bias & Std. & 0.100 & 0.050 \\
\hline 78 & RV & -0.28 & 1.19 & 0.1532 & 0.099 & TRV & 0.88 & 1.23 & 0.2958 & 0.1914 \\
& RV2 & -0.28 & 1.2 & 0.1532 & 0.0988 & TR2V & 0.39 & 1.1 & 0.1456 & 0.0698 \\
& pRV & -0.25 & 1.06 & 0.1088 & 0.0684 & R2V & 0.43 & 1.06 & 0.1386 & 0.0652 \\
& pRV2 & -0.27 & 1.16 & 0.1428 & 0.088 & R3V & 0.25 & 1.05 & 0.107 & 0.0518 \\
& cRV & -0.28 & 1.2 & 0.1562 & 0.1004 & R4V & 0.15 & 1.05 & 0.1004 & 0.0512 \\
\hline 390 & RV & -0.11 & 1.05 & 0.1166 & 0.065 & TRV & 1.87 & 1.5 & 0.5992 & 0.5086 \\
& RV2 & -0.11 & 1.06 & 0.1178 & 0.0658 & TR2V & 0.74 & 1.11 & 0.219 & 0.1302 \\
& pRV & -0.1 & 0.98 & 0.095 & 0.0464 & R2V & 0.97 & 1 & 0.2656 & 0.1678 \\
& pRV2 & -0.11 & 1.05 & 0.1174 & 0.0618 & R3V & 0.66 & 0.95 & 0.1604 & 0.0814 \\
& cRV & -0.11 & 1.1 & 0.133 & 0.0764 & R4V & 0.52 & 0.93 & 0.126 & 0.0618 \\
\hline 780 & RV & -0.08 & 1.02 & 0.104 & 0.0526 & TRV & 1.77 & 1.54 & 0.588 & 0.4902 \\
& RV2 & -0.08 & 1.02 & 0.1038 & 0.0522 & TR2V & 0.6 & 1.15 & 0.186 & 0.1104 \\
& pRV & -0.07 & 1.01 & 0.1024 & 0.0492 & R2V & 1.14 & 1 & 0.3338 & 0.2198 \\
& pRV2 & -0.08 & 1.05 & 0.1164 & 0.0574 & R3V & 0.74 & 0.94 & 0.1784 & 0.0968 \\
& cRV & -0.08 & 1.1 & 0.135 & 0.0728 & R4V & 0.58 & 0.93 & 0.137 & 0.0672 \\
\hline 4680 & RV & -0.03 & 0.98 & 0.0922 & 0.0482 & TRV & 0.46 & 1.79 & 0.2178 & 0.1462 \\
& RV2 & -0.03 & 0.98 & 0.091 & 0.0476 & TR2V & -0.02 & 1.55 & 0.1146 & 0.0656 \\
& pRV & -0.03 & 1.05 & 0.1152 & 0.0622 & R2V & 1.41 & 1.09 & 0.4264 & 0.3112 \\
& pRV2 & -0.03 & 1.05 & 0.1146 & 0.06 & R3V & 0.75 & 0.99 & 0.1982 & 0.1152 \\
& $\mathrm{cRV}$ & -0.03 & 1.14 & 0.1478 & 0.0822 & R4V & 0.54 & 0.97 & 0.1472 & 0.0772 \\
\hline
\end{tabular}


Table 18: Model (BNSCJ) with $\rho=-0.2$ corresponding to the jump part of the QV being $4.7 \%$.

\begin{tabular}{rrrrrrrrrrr}
\hline M & R.V.M. & Bias & Std. & 0.100 & 0.050 & R.V.M. & Bias & Std. & 0.100 & 0.050 \\
\hline 78 & RV & -0.25 & 1.18 & 0.144 & 0.0944 & TRV & -0.27 & 1.19 & 0.1494 & 0.0986 \\
& RV2 & -0.25 & 1.18 & 0.1444 & 0.0946 & TR2V & -0.38 & 1.19 & 0.1466 & 0.0976 \\
& pRV & -0.23 & 1.08 & 0.1184 & 0.073 & R2V & -0.33 & 1.17 & 0.138 & 0.0908 \\
& pRV2 & -0.25 & 1.15 & 0.1392 & 0.088 & R3V & -0.4 & 1.16 & 0.1442 & 0.0962 \\
& cRV & -0.25 & 1.19 & 0.1474 & 0.0968 & R4V & -0.44 & 1.14 & 0.147 & 0.0938 \\
\hline 390 & RV & -0.1 & 1.03 & 0.111 & 0.063 & TRV & -0.16 & 1.13 & 0.124 & 0.0758 \\
& RV2 & -0.1 & 1.03 & 0.1104 & 0.0628 & TR2V & -0.19 & 1.11 & 0.1218 & 0.071 \\
& pRV & -0.1 & 1 & 0.101 & 0.0554 & R2V & -0.1 & 1.03 & 0.1096 & 0.0588 \\
& pRV2 & -0.1 & 1.02 & 0.109 & 0.0616 & R3V & -0.14 & 1.03 & 0.113 & 0.0604 \\
& cRV & -0.1 & 1.04 & 0.1132 & 0.0654 & R4V & -0.17 & 1.03 & 0.1092 & 0.0614 \\
\hline 780 & RV & -0.07 & 1.01 & 0.107 & 0.055 & TRV & -0.15 & 1.21 & 0.1248 & 0.0712 \\
& RV2 & -0.07 & 1.01 & 0.1066 & 0.0546 & TR2V & -0.18 & 1.17 & 0.1214 & 0.0666 \\
& pRV & -0.07 & 0.99 & 0.0974 & 0.0494 & R2V & -0.06 & 1.01 & 0.1032 & 0.0498 \\
& pRV2 & -0.07 & 1 & 0.1036 & 0.054 & R3V & -0.1 & 1 & 0.1014 & 0.0526 \\
& cRV & -0.07 & 1.02 & 0.1084 & 0.0566 & R4V & -0.12 & 1 & 0.1002 & 0.0512 \\
\hline 4680 & RV & -0.04 & 1 & 0.0976 & 0.0502 & TRV & -0.23 & 1.76 & 0.1262 & 0.0762 \\
& RV2 & -0.04 & 0.99 & 0.097 & 0.0502 & TR2V & -0.21 & 1.62 & 0.1218 & 0.0778 \\
& pRV & -0.04 & 0.99 & 0.0974 & 0.0496 & R2V & 0 & 0.99 & 0.099 & 0.0536 \\
& pRV2 & -0.04 & 1 & 0.098 & 0.0506 & R3V & -0.01 & 0.99 & 0.0982 & 0.0546 \\
& cRV & -0.04 & 1.01 & 0.1002 & 0.0522 & R4V & -0.02 & 0.99 & 0.0984 & 0.0516 \\
\hline & & & & & & & & & &
\end{tabular}


Table 19: Model (BNSCJ) with $\rho=-0.4$ corresponding to the jump part of the QV being $16.7 \%$.

\begin{tabular}{rrrrrrrrrrr}
\hline M & R.V.M. & Bias & Std. & 0.100 & 0.050 & R.V.M. & Bias & Std. & 0.100 & 0.050 \\
\hline 78 & RV & -0.25 & 1.2 & 0.1452 & 0.0958 & TRV & -0.23 & 1.18 & 0.1446 & 0.0954 \\
& RV2 & -0.25 & 1.2 & 0.1444 & 0.0954 & TR2V & -0.35 & 1.17 & 0.1384 & 0.0962 \\
& pRV & -0.23 & 1.11 & 0.12 & 0.0742 & R2V & -0.28 & 1.17 & 0.1324 & 0.0882 \\
& pRV2 & -0.25 & 1.18 & 0.1386 & 0.0884 & R3V & -0.36 & 1.15 & 0.1376 & 0.0908 \\
& cRV & -0.25 & 1.23 & 0.1494 & 0.0996 & R4V & -0.41 & 1.13 & 0.1428 & 0.0914 \\
\hline 390 & RV & -0.1 & 1.03 & 0.1126 & 0.063 & TRV & -0.12 & 1.12 & 0.123 & 0.0726 \\
& RV2 & -0.1 & 1.03 & 0.1118 & 0.0626 & TR2V & -0.17 & 1.1 & 0.1194 & 0.0692 \\
& pRV & -0.09 & 1.01 & 0.1052 & 0.0572 & R2V & -0.05 & 1.04 & 0.1102 & 0.0588 \\
& pRV2 & -0.1 & 1.03 & 0.1114 & 0.0616 & R3V & -0.11 & 1.03 & 0.1098 & 0.0576 \\
& cRV & -0.1 & 1.06 & 0.1192 & 0.0688 & R4V & -0.14 & 1.02 & 0.1094 & 0.059 \\
\hline 780 & RV & -0.07 & 1.01 & 0.1082 & 0.055 & TRV & -0.12 & 1.19 & 0.1258 & 0.0718 \\
& RV2 & -0.07 & 1.01 & 0.1076 & 0.0536 & TR2V & -0.16 & 1.15 & 0.1176 & 0.066 \\
& pRV & -0.07 & 1 & 0.1014 & 0.0514 & R2V & -0.01 & 1.01 & 0.1044 & 0.0508 \\
& pRV2 & -0.07 & 1.01 & 0.1068 & 0.054 & R3V & -0.06 & 1 & 0.1004 & 0.0508 \\
& cRV & -0.07 & 1.04 & 0.115 & 0.0602 & R4V & -0.09 & 0.99 & 0.0998 & 0.0496 \\
\hline 4680 & RV & -0.04 & 1 & 0.0968 & 0.0498 & TRV & -0.2 & 1.72 & 0.1248 & 0.076 \\
& RV2 & -0.04 & 0.99 & 0.0962 & 0.0496 & TR2V & -0.2 & 1.58 & 0.1206 & 0.0766 \\
& pRV & -0.04 & 1 & 0.0998 & 0.0514 & R2V & 0.07 & 1.01 & 0.1064 & 0.0584 \\
& pRV2 & -0.04 & 1 & 0.0984 & 0.0512 & R3V & 0.01 & 0.99 & 0.0982 & 0.0526 \\
& cRV & -0.04 & 1.02 & 0.1058 & 0.0562 & R4V & 0 & 0.98 & 0.0982 & 0.0508 \\
\hline
\end{tabular}



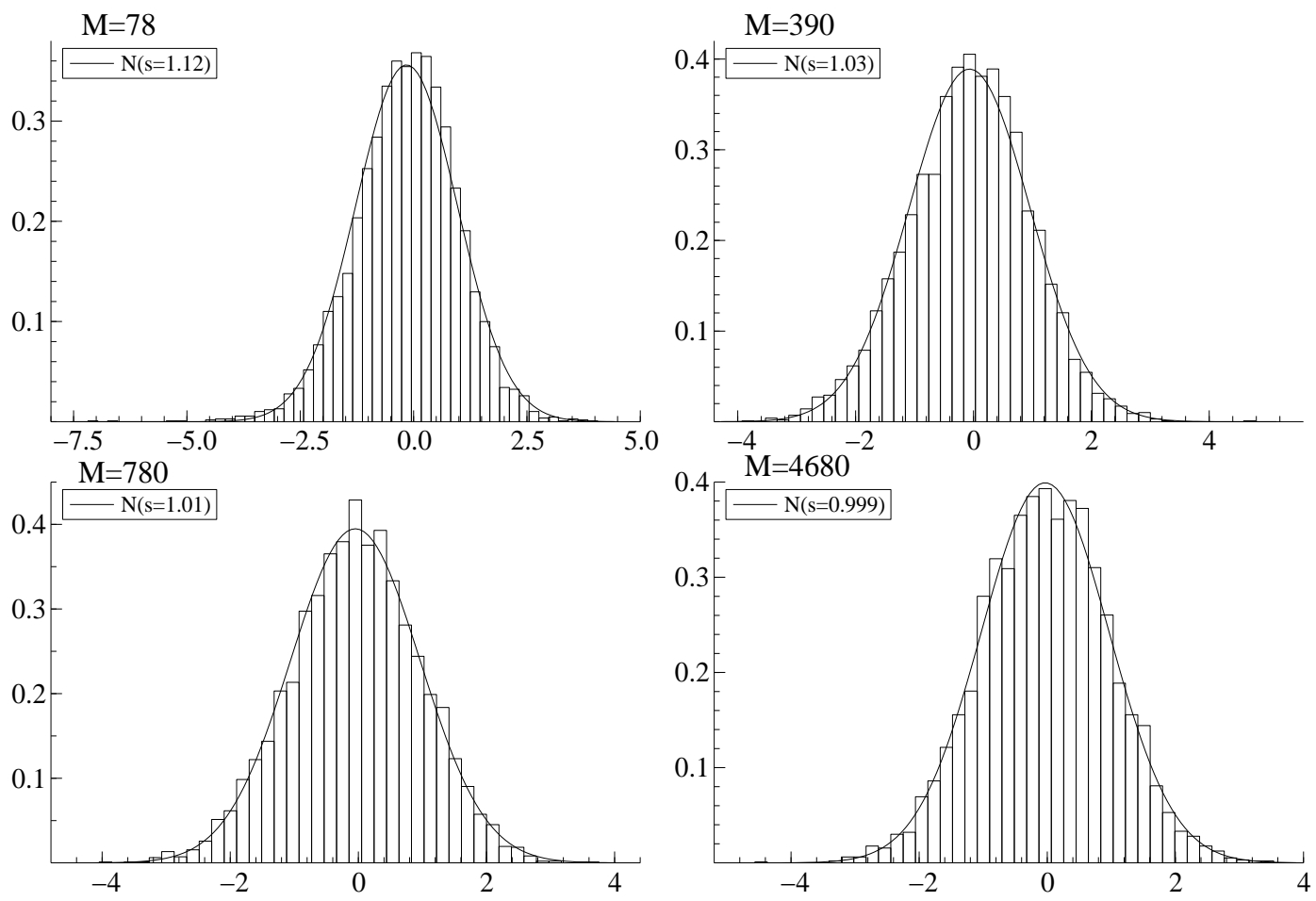

Figure 5: Empirical density of the logarithmic transform of centered and scaled realised variance (denoted by RV in the tables) for the model BNSCJ with $\rho=-0.4$. 


\section{References}

Ait-Sahalia, Y. \& Jacod, J. (2009a), 'Estimating the degree of activity of jumps in high frequency data', Annals of Statistics 37(5A), 2202-2244.

Aït-Sahalia, Y. \& Jacod, J. (2009b), 'Testing for jumps in a discretely observed process', Annals of Statistics 37(1), 184-222.

Aït-Sahalia, Y. \& Jacod, J. (2010), 'Is Brownian motion necessary to model high frequency data?', Annals of Statistics . Forthcoming.

Aït-Sahalia, Y. \& Kimmel, R. (2007), 'Maximum likelihood estimation of stochastic volatility models', Journal of Financial Economics 83(2), 413 - 452.

Andersen, T. G., Bollerslev, T. \& Diebold, F. X. (2007), Parametric and nonparametric measurement of volatility, Amsterdam: North Holland. Forthcoming.

Andersen, T. G., Bollerslev, T., Diebold, F. X. \& Ebens, H. (2001), 'The distribution of realized stock return volatility', Journal of Financial Economics 61, 43-76.

Andersen, T. G., Bollerslev, T., Diebold, F. X. \& Labys, P. (2001), 'The distribution of exchange rate volatility', Journal of the American Statistical Association 96, 42-55. Correction published in 2003, Volume 98, Page 501.

Bandi, F. M. \& Renò, R. (2010), Nonparametric stochastic volatility. Available at SSRN: http://ssrn.com/abstract=1158438.

Barndorff-Nielsen, O. E., Hansen, P. R., Lunde, A. \& Shephard, N. (2008a), 'Designing realized kernels to measure the ex post variation of equity prices in the presence of noise', Econometrica 76(6), 1481-1536.

Barndorff-Nielsen, O. E., Hansen, P. R., Lunde, A. \& Shephard, N. (2008b), Multivariate realised kernels: consistent positive semidefinite estimators of the covariation of equity prices with noise and non-synchronous trading. CREATES working paper 08-63.

Barndorff-Nielsen, O. E. \& Shephard, N. (2001), 'Non-Gaussian Ornstein-Uhlenbeck-based models and some of their uses in financial economics (with discussion)', Journal of the Royal Statistical Society Series B 63, 167-241.

Barndorff-Nielsen, O. E. \& Shephard, N. (2002), 'Econometric analysis of realised volatility and its use in estimating stochastic volatility models', Journal of the Royal Statistical Society B 64, 253 280.

Barndorff-Nielsen, O. E. \& Shephard, N. (2004a), 'Econometric analysis of realised covariation: high frequency covariance, regression and correlation in financial economics', Econometrica 72, 885925 .

Barndorff-Nielsen, O. E. \& Shephard, N. (2004b), 'Power and bipower variation with stochastic volatility and jumps (with discussion)', Journal of Financial Econometrics 2, 1-48.

Barndorff-Nielsen, O. E. \& Shephard, N. (2006), 'Econometrics of testing for jumps in financial economics using bipower variation', Journal or Financial Econometrics 4, 1-30. 
Barndorff-Nielsen, O. E. \& Shephard, N. (2007), Variation, jumps, market frictions and high frequency data in financial econometrics, in R. Blundell, P. Torsten \& W. K. Newey, eds, 'Advances in Economics and Econometrics. Theory and Applications', Vol. III of Ninth World Congress, Econometric Society Monographs, Cambridge University Press.

Barndorff-Nielsen, O. E., Shephard, N. \& Winkel, M. (2005), Limit theorems for multipower variation in the presence of jumps, Economics Papers 2005-W07, Economics Group, Nuffield College, University of Oxford.

Black, F. (1976), 'Studies of stock market volatility changes', Proceedings of the Business andEconomic Statistics Section, American Statistical Association pp. 177-181.

Blumenthal, R. \& Getoor, R. (1961), 'Sample functions of stochastic processes with independent increments', Journal of Mathematics and Mechanics 10, 493-516.

Cont, R. \& Mancini, C. (2007), Nonparametric tests for analyzing the fine structure of price fluctuations, Technical report, Columbia University Financial Engineering Report No. 2007-13. Available at SSRN: http://ssrn.com/abstract=1081491.

Corsi, F., Pirino, D. \& Renò, R. (2010), 'Threshold bipower variation and the impact of jumps on volatility forecasting', Journal of Econometrics . Accepted manuscript, DOI: 10.1016/j.jeconom.2010.07.008.

Dette, H., Podolskij, M. \& Vetter, M. (2006), 'Estimation of integrated volatility in continuous time financial models with applications to goodness-of-fit testing', Scandinavian Journal of Statistics 33, 259-278.

Foster, D. P. \& Nelson, D. B. (1996), 'Continuous record asymptotics for rolling sample variance estimators', Econometrica 64(1), 139-174.

Hansen, P. R. \& Lunde, A. (2006), 'Realized variance and market microstructure noise', Journal of Business and Economic Statistics 24, 127-218.

Huang, X. \& Tauchen, G. (2005), 'The Relative Contribution of Jumps to Total Price Variance', Journal of Financial Econometrics 3(4), 456-499.

Jacod, J. (1994), Limit of random measures associated with the increments of a Brownian semimartingale. Preprint number 120, Laboratoire de Probabilitiés, Université Pierre et Marie Curie, Paris.

Jacod, J. (2004), 'The Euler scheme for Lévy driven stochastic differential equations: Limit theorems', The Annals of Probability 32(3A), 1830 - 1872.

Jacod, J. (2006), Asymptotic properties of realized power variations and related functionals of semimartingales: Multipower variation. Université P. et M. Curie (Paris 6): Unpublished paper.

Jacod, J. (2008), 'Asymptotic properties of realized power variations and related functionals of semimartingales', Stochastic processes and their applications 118, 517-559.

Jacod, J. \& Protter, P. (1998), 'Asymptotic error distribution for the Euler method for stochastic differential equations', The Annals of Probability 26(1), 267 - 307.

Jacod, J. \& Todorov, V. (2010), 'Do price and volatility jump together?', Annals of Applied Probability . Forthcoming. 
Kristensen, D. (2010), 'Nonparametric filtering of the realised spot volatility: A kernel-based approach', Econometric Theory 26, 60-93.

Lee, S. S. \& Mykland, P. A. (2008), 'Jumps in financial markets: A new nonparametric test and jump dynamics', Review of Financial Studies 21, 2535-2563.

Mancini, C. (2001), 'Disentangling the jumps of the diffusion in a geometric jumping brownian motion', G. dell'Inst. Ital. degli Attuari LXIV, 19-47.

Mancini, C. (2009), 'Non-parametric threshold estimation for models with stochastic diffusion coefficient and jumps', Scandinavian Journal of Statistics 36, 270-296.

Mancini, C. \& Gobbi, F. (2010), Identifying the Brownian covariation from the co-jumps given discrete observations, DiMaD Working Papers 2010-05, Dipartimento di Matematica per le Decisioni, Universita' degli Studi di Firenze.

URL: http://ideas.repec.org/p/flo/wpaper/2010-05.html

Nelson, D. B. (1991), 'Conditional heteroscedasticity in asset returns: a new approach', Econometrica 59(2), 347-370.

Podolskij, M. \& Vetter, M. (2009), 'Estimation of volatility functionals in the simultaneous presence of microstructure noise and jumps', Bernoulli 15(3), 634-658.

Protter, P. E. (2004), Stochastic Integration and Differential Equations, second edn, Springer, London.

Rényi, A. (1963), 'On stable sequences of events', Sankhya-: The Indian Journal of Statistics, Series A 25(3), 293-302.

Rosinski, J. (2001), Series representations of Lévy processes from the perspective of point processes, in O. Barndorff-Nielsen, T. Mikosch \& S. Resnick, eds, 'Levy Processes Theory and Applications', Birkhäuser, Boston.

Todorov, V. \& Tauchen, G. (2010), 'Limit theorems for power variations of pure-jump processes with application to activity estimation', Annals of Applied Probability. Accepted manuscript.

Veraart, A. E. D. (2010), 'Inference for the jump part of quadratic variation of Itô semimartingales', Econometric Theory 26(2), 331-368.

Veraart, A. E. D. \& Veraart, L. A. M. (2010), 'Stochastic volatility and stochastic leverage', Annals of Finance . Online First: DOI: 10.1007/s10436-010-0157-3.

Vetter, M. (2010), 'Limit theorems for bipower variation of semimartingales', Stochastic Processes and their Applications 120, 22-38.

Woerner, J. H. C. (2006), Power and multipower variation: inference for high frequency data, in A. N. Shiryaev, M. R. Grossinho, P. E. Oliveira \& M. L. Esquível, eds, 'Stochastic Finance', Springer US, pp. 343-364.

Zhang, L. (2006), 'Efficient estimation of stochastic volatility using noisy observations: A multi-scale approach', Bernoulli 12(6), 1019-1043. 
Zhang, L., Mykland, P. A. \& Aït-Sahalia, Y. (2005), 'A tale of two time scales: determining integrated volatility with noisy high-frequency data', Journal of the American Statistical Association 100, 1394-1411.

Zhou, H. (2003), 'Itô conditional moment generator and the estimation of short-rate processes', Journal of Financial Econometrics 1(2), 250-271. 
2010-52: Christos Ntantamis: Detecting Structural Breaks using Hidden Markov Models

2010-53: Christos Ntantamis: Detecting Housing Submarkets using Unsupervised Learning of Finite Mixture Models

2010-54: Stefan Holst Bache: Minimax Regression Quantiles

2010-55: Nektarios Aslanidis and Charlotte Christiansen: Sign and Quantiles of the Realized Stock-Bond Correlation

2010-56: Anders Bredahl Kock: Oracle Efficient Variable Selection in Random and Fixed Effects Panel Data Models

2010-57: Charlotte Christiansen, J uanna Schröter J oensen and J esper Rangvid: The Effects of Marriage and Divorce on Financial Investments: Learning to Love or Hate Risk?

2010-58: Charlotte Christiansen, Maik Schmeling and Andreas Schrimpf: A Comprehensive Look at Financial Volatility Prediction by Economic Variables

2010-59: J ames G. MacKinnon and Morten Ørregaard Nielsen: Numerical distribution functions of fractional unit root and cointegration tests

2010-60: Bent J esper Christensen and Paolo Santucci de Magistris: Level Shifts in Volatility and the Implied-Realized Volatility Relation

2010-61: Christian Bach and Bent J esper Christensen: Latent Integrated Stochastic Volatility, Realized Volatility, and Implied Volatility: A State Space Approach

2010-62: Bent J esper Christensen and Malene Kallestrup Lamb: The Impact of Health Changes on Labor Supply: Evidence from Merged Data on Individual Objective Medical Diagnosis Codes and Early Retirement Behavior

2010-63: Martin M. Andreasen: How Non-Gaussian Shocks Affect Risk Premia in Non-Linear DSGE Models

2010-64: Tim Bollerslev and Viktor Todorov: J ump Tails, Extreme Dependencies, and the Distribution of Stock Returns

2010-65: Almut E. D. Veraart: How precise is the finite sample approximation of the asymptotic distribution of realised variation measures in the presence of jumps? 